\title{
A New Radioactive Decay Mode, True Ternary Fission, the Decay of Heavy Nuclei Into Three Comparable Fragments.
}

\author{
W. von Oertzen ${ }^{1}$ A. K. Nasirov ${ }^{2}$ \\ 1 Helmholtz-Zentrum Berlin, Hahn-Meitner Platz 1, 14109 Berlin, Germany, and Fachbereich Physik Freie Universitaet Berlin, \\ 2 Bogoliubov Laboratory of Theoretical Physics, JINR, Dubna 141980 Russia, Institute of Nuclear Physics, Ulugbek, \\ Tashkent,100214, Uzbekistan
}

Received: date / Revised version: date

\begin{abstract}
The ternary cluster decay of heavy nuclei (e.g. in spontaneous fission of ${ }^{252} \mathrm{Cf}(\mathrm{sf}, \mathrm{fff})$ ), has been observed in several experiments with binary coincidences between two fragments using detector telescopes (the FOBOS-detectors) with very large solid angles and placed on the opposite sides from the source of fissioning nuclei. The binary coincidences at a relative angle of $180^{\circ} \mathrm{deg}$. correspond to binary fission or to the decay into three cluster fragments by registration of two of them in coincidences with missing nuclei of different masses (e.g. ${ }^{132} \mathrm{Sn},{ }^{52-48} \mathrm{Ca},{ }^{68-72} \mathrm{Ni}$ ). This marks a new step in the physics of fission-phenomena of heavy nuclei. The new decay mode has been observed more then 10 years ago by the FOBOS group in JINR (Dubna) Russia. These experimental results for the collinear cluster tripartition (CCT), refer to the decay into three clusters of comparable masses. In the present work we discuss the various aspects of this ternary fission (FFF) mode, with different mass partitions. The question of collinearity is analysed on the basis of recent publications. Further insight into the possible decay modes is obtained by the discussion of the path towards larger deformation, towards hyper-deformation and by inspecting details of the potential energy surfaces (PES). The PES is determined as the total sum of the masses, including the shell effects, which enter with $Q_{g g g}$, the three-body Q-value for the separation into three fragments, and the total interaction (nuclear and Coulomb) between all three nuclei. In the path towards the extremely deformed states leading to ternary fission, the concept of deformed shells is most important. At the scission configuration the phase space determined by the PES's leads to the final mass distributions. The possibility of formation of fragments of almost equal size $\left(Z_{i}=32,34,32\right.$, for $\left.Z=98\right)$ and the observation of several other fission modes in the same system can be predicted by the PES. The PES's show pronounced minima and valleys, where the phase space for the decay reaches maximum values, namely for several mass/charge combinations of ternary fragments, which correspond to a variety of collinear ternary fission (multi-modal) decays. The case of the decay of ${ }^{252} \mathrm{Cf}$ (sf,fff) turns out to be unique due to the presence of deformed shells in the total system and of closed shells in all three nuclei in the decay.
\end{abstract}

PACS. 23.70. + j Heavy-particle decay - 25.85.Ca Spontaneous fission - 25.85.Ec Neutron-induced fission

\section{Introduction, Binary Fission}

Binary fission of nuclei after irradiation of Uranium with slow neutrons has been observed by Otto Hahn and Fritz Strassman in 1938, 1]. The fragments of Barium (charge $Z$ $=56$ ) have been identified by methods of radio-chemistry. This group from Berlin, together with Lise Meitner, who had to escape from Nazi-Germany in 1938, had previously studied a variety of nuclear processes induced by neutrons. Actually L. Meitner urged Hahn repeatedly to study in more detail the reaction products observed from the irradiation of Uranium-foils with neutrons. Different groups, in particular Fermi (see Ref. 2] ) had published results, where new radio-activities have been observed and attributed to new elements heavier then Uranium. Very controversial results of other groups followed in the 1936-
38 's. Fermi actually received the Nobel-price for this work of finding new elements (incorrect!) in 1938.

Lise Meitner had worked together with O. Hahn in Berlin for many years. L. Meitner as an Austrian citizen of Jewish origin, had to escape from Nazi-Germany, she went to Stockholm, Sweden in the fall of 1938, when Hitler invaded Austria ("Anschluss"). Due to these circumstances she could not participate in the final phases of the work of her colleagues in Berlin by O. Hahn and F. Strassman. They published their results without Lise Meitner, in Naturwissentschaften, 27 p11, (1939). However, O. Hahn was eager to tell L.Meitner about his final result, already earlier and with an exchange of letters in the fall of 1938 and in Jan. 1939 between Stockholm, Copenhagen and Berlin. Lise Meitner was able to produce a paper with her nephew Otto Frisch (at that time in Copenhagen) 
which gave the first interpretation of the results of fission: L. Meitner und O. Frisch, Ref. [3]: "Disintegration of Uranium by Neutrons, a new type of Nuclear Reaction". Frisch actually introduced here the concept/expression of "fission" for the splitting of Uranium-nuclei. Meitner and Frisch were the first to realize the very large energy-release involved in fission: $>200 \mathrm{MeV}$, a very large (enormous!) value for the energy-release in a nuclear decay, at that time such a high value has never been observed before. With this result an avalanche of publications started in 1939. For their first publication in the Nature-journal, the draft-paper had been presented by Frisch to Niels Bohr (one of the giants of atomic/nuclear physics at that time) in January 1939 in Copenhagen, before his departure to the USA. Reading their draft, Bohr exclaimed "what fools we have been"! In fact the result could have been obtained many years before, using the by that time well known formula for the mass(energy) of nuclei with the liquid-drop model.

The liquid drop model for the calculation of the binding energy of nuclei has been developed in the years 1933-35 . The total binding energy of nuclei is illustrated in Fig. 1, as function of the binding energy per nucleon. From this figure we deduce that the values reach their maximum for nuclear masses of $A=(60-80)$. This fact was of course also well known by most nuclear physicists in 1938. The heavy fragments (Kr, Ba) with their high electric charges and large values of the kinetic energy released in the fission process give rise to very large signals in the ionisation chambers used at that time (often misinterpreted as "failures" of the system). Soon after the news spread in the beginning of the year 1939, several groups were able to register directly the fission fragments with ionisation chambers, because they found large signals due to the large charges of the fragments $(\mathrm{Ba}, \mathrm{Z}=56$ and $\mathrm{Kr}$, $\mathrm{Z}=36$ ). Arriving in the USA in the beginning of 1939 in Princeton Niels Bohr worked with J. Wheeler and they produced within six month a monumental publication, title: "The Mechanism of Nuclear Fission" in: Physical Review Vol. 56 (1939) 426 4. Still today a bible for nuclear physicists studying fission. Nuclear fission is a fascinating object of study, because it involves the interplay of macroscopic and microscopic quantum properties of nuclear matter. For the following decades and years many groups have studied binary fission in detail, for an intermediate stage of our knowledge we can look into the proceedings of conferences for the 50'ties anniversary for the discovery of fission:

Four conferences on fission have been devoted in 1989 to this anniversary: 50 Years of Nuclear Fission [5], in the Journal: Nuclear Physics A 502 (1989), 1-639, eds. D. Hilscher, H. Krappe and W. von Oertzen. Further: 50 years with nuclear fission, National Academy of Science, Washington D.C., USA, and National Institute of Standards and Technology, Gaithersburg, Maryland USA Ref. 6]. Another conference has been held in Leningrad (Soviet Union) October 16-20, 1989: Proceedings ed. L.W. Drabchinski, Fission of Nuclei, 50 Years, Chlopin Radium Institute, 1992, Vol. 1 and 2 [7], (dominantly in Russian).
The study of binary fission over the last decades has produced a large body of experimental results with emphasis on a detailed description of several parameters such as the mass and charge distribution of fission fragments and their kinetic energies. For the heavier nuclei the fission decay may become the dominant decay channel. An important step for the understanding of the fission process is the appearance of shell structures in heavy deformed nuclei, as introduced by Strutinsky 8,9 . The shell effects play decisive role in the yield of fragments, which is varying as function of charge and neutron number. The variation of the mass distribution of fission fragments with the given proton and neutron numbers has been found to depend mainly on the spherical and deformed shells. These phenomena are described with a large variety of theoretical approaches, many of these have been put forward during the last decades. In the recent book by $\mathrm{H}$. Krappe and K. Pomorski [10] many of these established approaches are described.

For an earlier overview on scientific aspects of (binary) fission we suggest the book edited by C. Wagemans 1991: "The Nuclear Fission Process" [1], covering all important aspects of this process. The fact which dominates the mass-distributions of the fission fragments are the shell effects for the charges $\mathrm{Z}=20,28,50$, and for the neutrons with $\mathrm{N}=20,28,50$ and 82 . Thus the mass distribution of binary fission is asymmetric as shown in Fig. 2 .

In addition this figure (from Ref. [12]) shows the yield for light fragments with masses from $A=4$ up to $A=$ 30 , observed in coincidence with binary fission channels. These are typically emitted perpendicular to the fission axis, determined by the vectors of the two heavy fragments. These events usually have been defined as "ternary fission" 12. The "true ternary fission" of heavy nuclei discussed in the present survey, has been predicted many times in theoretical works since the early 1950-60'ties, a decay with an increasing probability for increasing total charge of the nuclei.

\section{Binary and Ternary Fission}

\subsection{General considerations, shell effects, hyper-deformation, sequential decay}

Fission involves a rearrangement of nucleons in a collective macroscopic motion (evolution in shapes) towards an elongated, deformed structure with energies dominated by the liquid drop aspects and the quantal properties of nuclei, the shell effects. These aspects have been described earlier, as an example we cite the work of Swiatecki [13] and of Diehl and Greiner [14. In the first it has been indicated that apart of binary decays, ternary (and multiple) decays are energetically possible in heavy nuclei. The probability for the decay depends on the barriers for the individual combinations of fragments and on the phase space in these decays. Quite important is the variation of fission properties in dependence on the total mass and charge of the fissioning nucleus and the appearance of shells, particularly for protons. For the heavier nuclei 


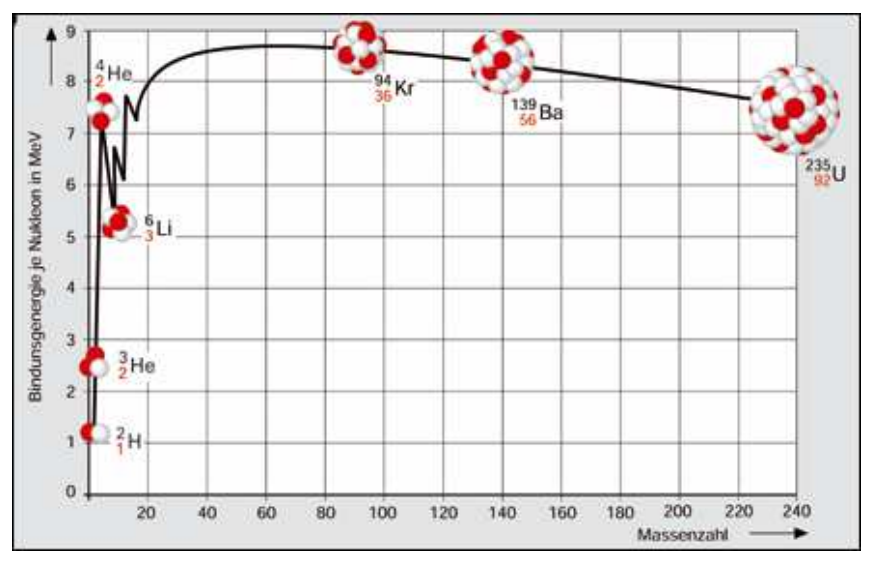

Fig. 1. The binding energies per nucleon $\left(E_{B} /\right.$ nucleon $)$ in nuclei as function of mass $A$. The maximum is reached for masses with $\mathrm{A}=60-110$. Ternary fission of heavy nuclei into three fragments with these masses is possible. The liquid drop model describes the variation of the total binding energy as shown in the figure.

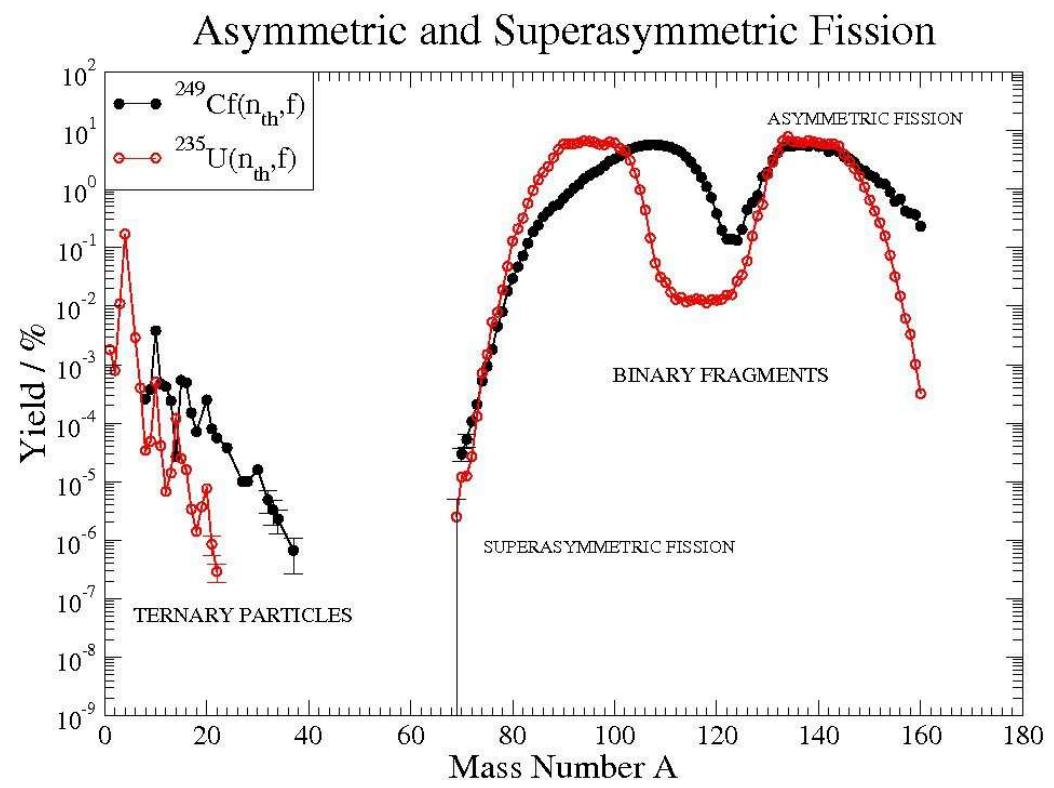

Fig. 2. Mass distribution of binary fission fragments from the spontaneous decay of ${ }^{252} \mathrm{Cf}(\mathrm{sf})$, as function of fragment mass. The shell model explains the two hump distribution with enhanced yields for masses around $A=80-110$ and $A=120-140$. The left side of the figure shows the yields of lighter third fragments in coincidence with two binary fission fragments, for two systems as indicated, from Ref. [12].

the fission decay may become the dominant decay channel. As already mentioned an important step for the understanding of the fission process is the appearance of shell structures in heavy deformed nuclei, as introduced by Strutinsky [8, 9]. This very unique approach has been essential for nuclear structure studies by describing the super - and hyper-deformed shapes. Further super- and hyper-deformation played an important role in the more recent studies in $\gamma$-spectroscopy of high spin states 15 . The hyper-deformed configurations at low spin show im- portant manifestations with the occurrence of fission isomers 16, 17, 18, which have been studied extensively 40 years ago $19,20,21$. Thus it appears that the ternary decay passes through hyper-deformed shapes of the fissioning nucleus (see Fig. 3).

In the following we list the factors governing the probabilities (the phase space) for these decays:

i) the details of the potential energy surface, the PES,

ii) its valleys and hills,

iii) the internal barriers at the two necks, 


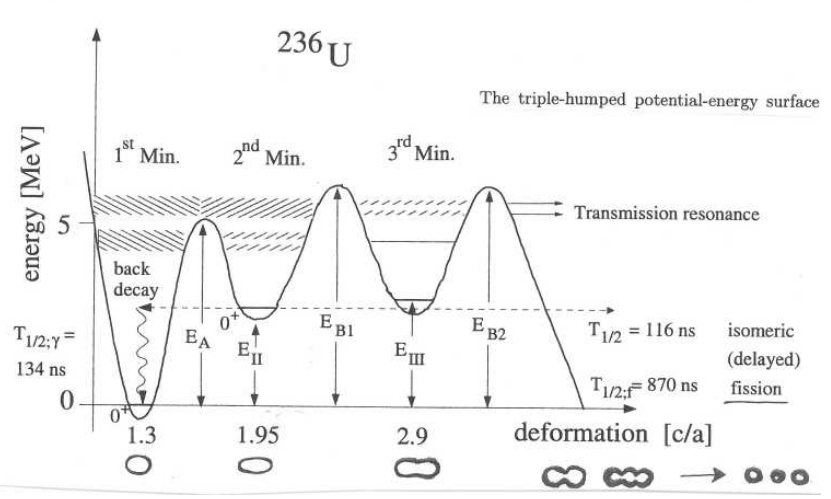

Fig. 3. The potential energy as function of deformation for a hyper-deformed nuclear shape, which shows the double humped fission barrier suggesting a path to the collinear ternary fission decay in ${ }^{236} \mathrm{U}$, adapted from Ref. 21.

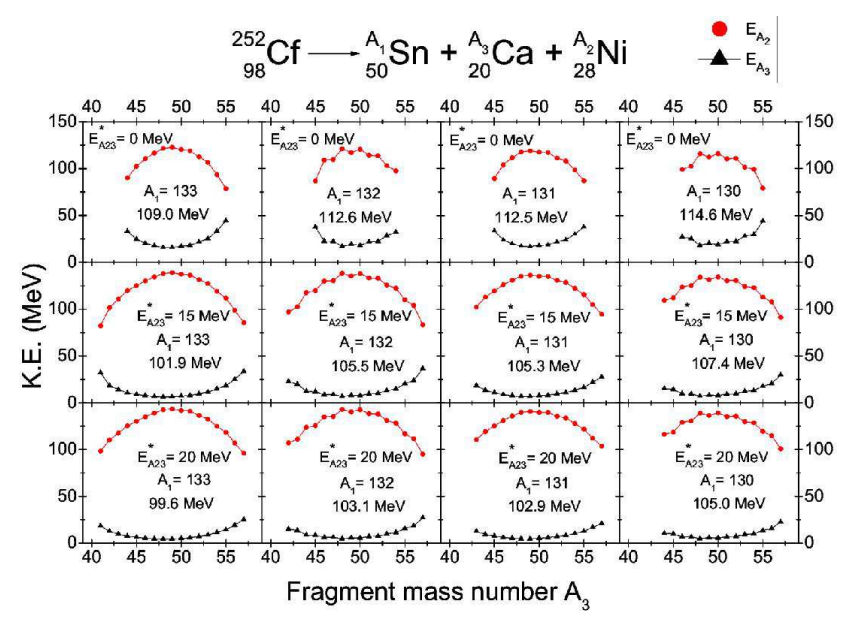

Fig. 4. Maximum values of the kinetic energies of fragments $A_{3}$ and $A_{2}$ in the model with sequential decay (as shown in Fig. 19p leading to ternary decays, (from Ref. 22]). A similar result has been obtained in Ref. 23

iv) the $Q_{g g g}$-values, the latter determining the kinetic energies and the number of possible fragment (isotope) combinations,

v) the excitation energy range in the individual fragments, vi) the momentum range for these,

vii) the number of excited states (or the density of states) in each of the fragments, the combinations consisting of 3 isotopes, and by

viii) the spin $(J)$-multiplicity (phase space factor $(2 J+1)$ ) in these excited states, with spins expected up to values of $(6-8)^{+}$.

More general considerations for a three-body decay have been analysed in Ref. 25. From this work we can state that the radioactive ternary fission decay, being allowed from $Q$-value considerations, will proceed sequen-

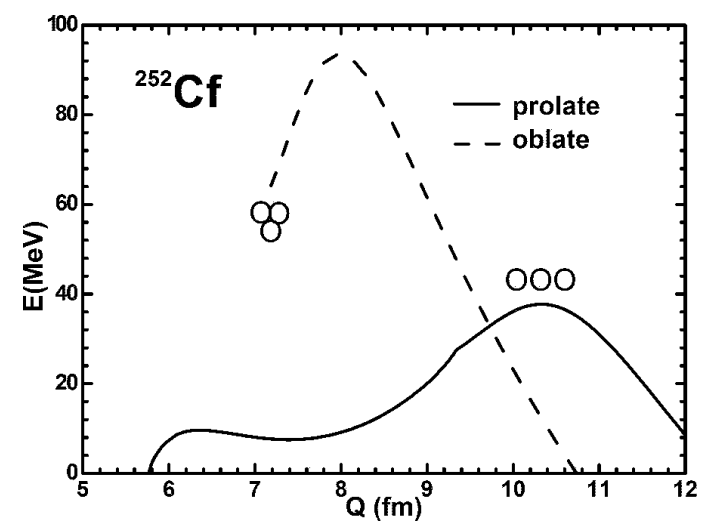

Fig. 5. Comparison of the total potential energies, dominated by the Coulomb interaction, of three fragments in an oblate configuration compared to a prolate (collinear) shape for three cluster decay, courtesy of Royer [24].

tially, because the two barriers and the phase space favour the individual steps, the first steps are determined by the lower barriers and their phase space. The first step usually will start with the higher probability and proceed with the lighter mass combination of fragments with a lower barrier and the higher energy balance, which has the larger preferred phase space. For this case of sequential decay we can calculate the kinetic energies of the ternary fission decay of ${ }^{252} \mathrm{Cf}$ (sf,fff) with the decay mode into the three fragments: ${ }^{70} \mathrm{Ni}+{ }^{48} \mathrm{Ca}+{ }^{132} \mathrm{Sn}$, which are most likely. The result is shown in Fig 4 and discussed further in sect 2.3 , and illustrated in Fig. 19. Actually in this simple model the kinetic energies of the outer fragments are high, the figure shows obtained maximum values, they are too high (as indicated in Ref. 26]) because no excitation energy of the fragments is considered.

An important approach is to consider the connection between ternary fission and extreme deformations, namely hyper-deformation, illustrated in Fig. 3. In this context the work of Brosa et al. [27] is of importance, in this work different fission paths are obtained, there extremely long ("super long") shapes with very large deformations have been predicted. Fission must be considered as a macroscopic rearrangement of all nucleons into a hyper-deformed shape with two necks. There will be two neck ruptures in a very short time sequence.

The term "ternary fission" has been used for binary fission accompanied by light particle (like $\alpha$-particles) emission. For such decays with a third light particle emitted perpendicular to the binary fission axis, compilations are available in Ref. 12. These must be considered to be emitted from an prolate-binary configuration with a neck, the corresponding yields decrease strongly (see Fig. 2) as function of increasing mass(charge) of the third particle, from Ref. 12. For the general survey of the possible ternary decays (FFF), and the discussion of the relative probabilities of oblate to prolate ternary fission, we must look into the Coulomb energy of the total system. In Fig. 5 we show 


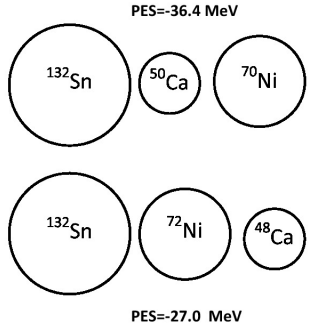

Fig. 6. The total potential energy of three fragments in a collinear configuration, it is smallest with the smaller fragment at the center. The collinear configuration dominates the three cluster decay.

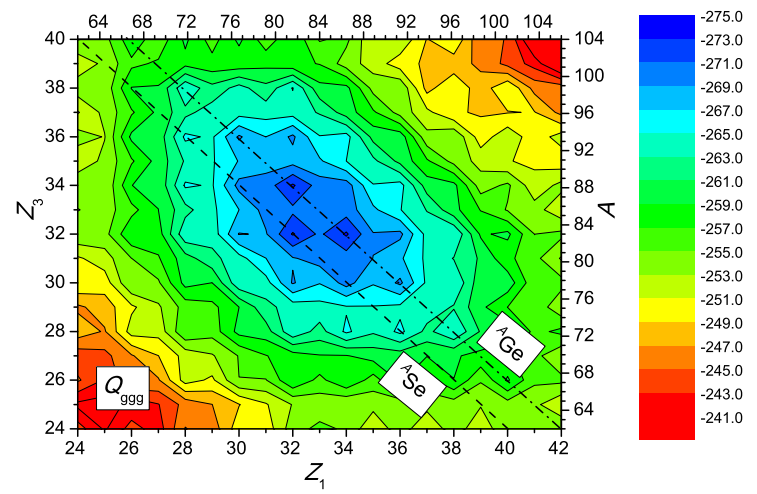

Fig. 7. The $Q_{g g g}$-values (-2n) for ${ }^{252} \mathrm{Cf}$, the ternary fragments with $Z_{i}=32-34$, correspond to the highest Q-values. the most symmetric decays, however, with the highest internal barriers.

the comparison, from Ref. 24, of the PES of an oblate versus a prolate arrangement of three equal (comparable) sized fragments, which can be related to the ternary decay of ${ }^{252} \mathrm{Cf}(\mathrm{fff})$.

Clearly the linear arrangement will be preferred. Further, the potential energies, actually the PES as a function of mass asymmetry, contain the most relevant quantities determining the phase space of the decays (discussed in detail later). The dominance of the Coulomb interaction points to distinct geometrical arrangements of the three clusters as shown in Fig. 5 and Fig. 6. The latter implies that the collinear arrangements with the smallest fragment in the center will have the largest phase space and therefore the highest probability.

The almost symmetric combination of three fragments gives maximal $Q_{g g g}$ values, as indicated in Fig. 7. however, as shown later, this mode has a very low yield, because the corresponding potential energy barriers are high, and the probability of its population is small, because the highest internal barriers occur, making the decay difficult from these configurations. Still the symmetric decays into three comparable fragments with the largest $Q$-value, can be extracted from the data (see sect. 4).

We have already introduced the concept of hyper deformation, which must be considered in the dynamical path towards a three fragment channel. In this the com-

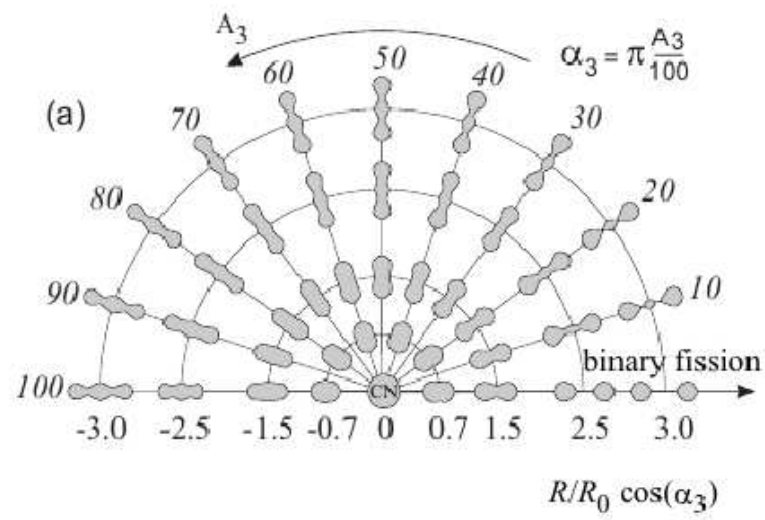

Fig. 8. The macroscopic geometry and coordinates chosen for the ternary fission study of Karpov in the three center-shell model. The coordinates are defined appropriate to the ternary decay, from Ref. 28. The formation of shapes reminiscent of super- and hyper-deformation in nuclei is observed. The radius of the spherical nucleus $(\mathrm{CN})$ is denoted as $\mathrm{R}_{0}$.

bination of macro- and micros-copic aspects of the potential energies as function of smoothly varying nuclear shapes become essential for the understanding of superand hyper-deformation. Related to this quite important and convincing results concerning the physical circumstances for ternary fission have been obtained in the work of Karpov [28. In this work the asymmetric three-center shell model is used, which is related to the two-center shell model, originally developed in 1972 by Greiner et.al. by the Frankfurt group [29]. We will see that the structure of this PES is dominated by the shell effects in all three fragments like in ${ }^{132} \mathrm{Sn},{ }^{48} \mathrm{Ca}$ and ${ }^{72} \mathrm{Ni}$ see Figs. 9, 10 and Fig. 11. In this work appropriate shapes and coordinates are introduced in order to describe the ternary decay, they are shown in Fig 8 .

Macroscopic potential energy (dashed line), shell correction (dotted line), and total macro-microscopical potential energy (solid line) of the ${ }^{252} \mathrm{Cf}$ nucleus corresponding to the ${ }^{132} \mathrm{Sn}+{ }^{48} \mathrm{Ca}+{ }^{72} \mathrm{Ni}$ ternary splitting.

In these calculations the favored fission path (dashed line in Fig. 11 passes through two barriers. The first barrier is higher the second barrier appears at rather large elongations(deformations). In the discussion of the results, the author analyses the macroscopic and microscopic features of the PES. The barriers are shown in Fig. 9. The second neck appears in the macroscopic calculation, with a very high second barrier (see Fig. 9), ternary fission is not possible with the liquid drop shapes. The inclusion of the shell effects in all three fragments produces the important effects: the fission barrier becomes doublehumped. The hyper-deformation appears (see Fig. 3), a feature giving rise to the fission-isomers in the actinides. The ternary fission barrier is reduced dramatically, the barriers exist in the elongation parameter (deformation) and in the mass asymmetry. Actually the binary fission is suppressed and the probability for the ternary decay increases. Apart from the case discussed, other channels will 


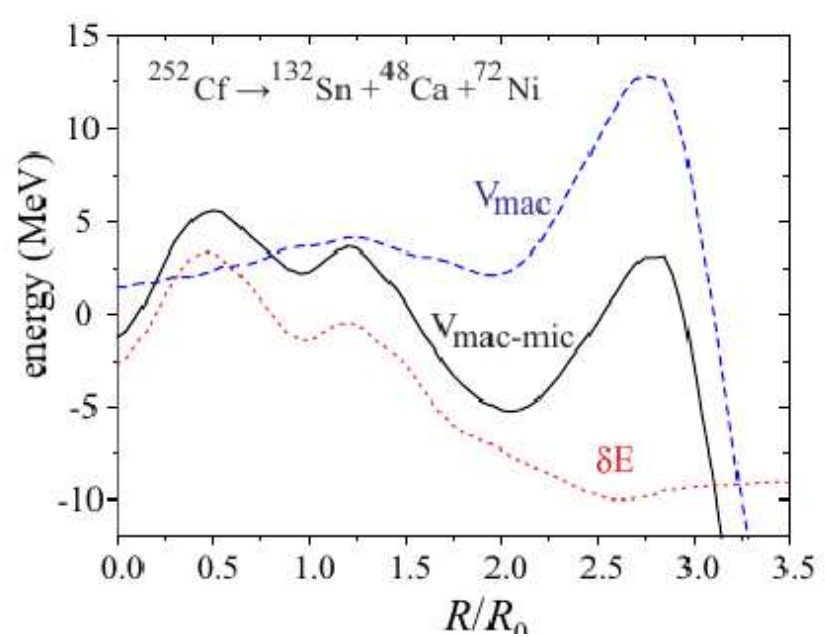

Fig. 9. The macroscopic (dashed line) potential energy of the three-center system as function of the reduced elongation. The shell correction ( $\delta E$, red dotted line), and the full macro-microscopic potential energy of ${ }^{252} \mathrm{Cf}$ with a shape corresponding to the splitting into $\left({ }^{132} \mathrm{Sn},{ }^{48} \mathrm{Ca}\right.$ and $\left.{ }^{72} \mathrm{Ni}\right)$, from Ref. 28. The third barrier (in the mac-mic approach) is strongly reduced.

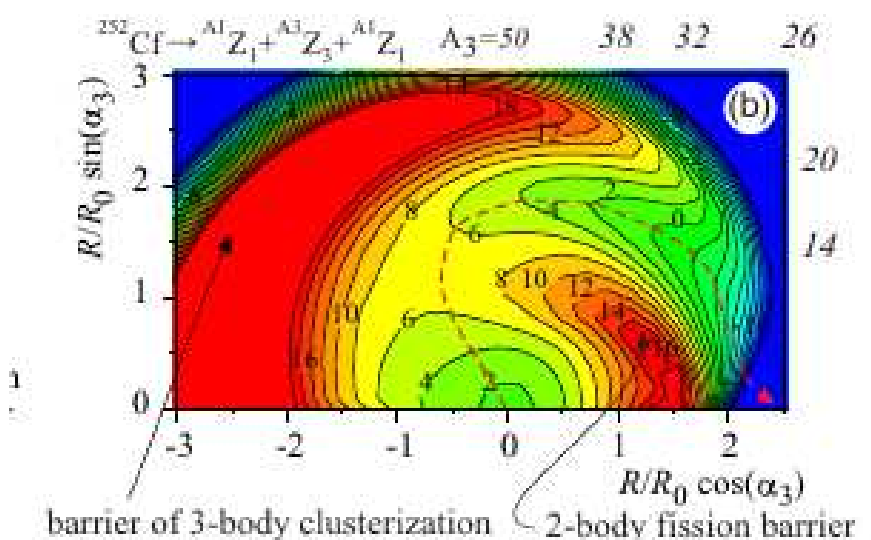

Fig. 10. The potential energy surface for ${ }^{252} \mathrm{Cf}$ as function of the elongation of the system, it shows the binary fission path, this figure is without the shell effects, see the next figure. Results obtained with the three-center shell model. The dominant decay is always with ${ }^{132} \mathrm{Sn}$. The approach also secribes (dashed line) the binary fission path, from Ref. 28].

be favored like ("tin-sulfur-germanium") and the combination ("nickel-oxygen-samarium"). The ternary decay of ${ }^{252} \mathrm{Cf}$ observed in the experiments is due to the unique configurations with deformed shells and the shells in the fragments.

\subsection{Potential Energy Surfaces, Different Ternary Decays}

With the evolution of the nuclear shape towards the ternary mass split through a hyper-deformed shape with the for-

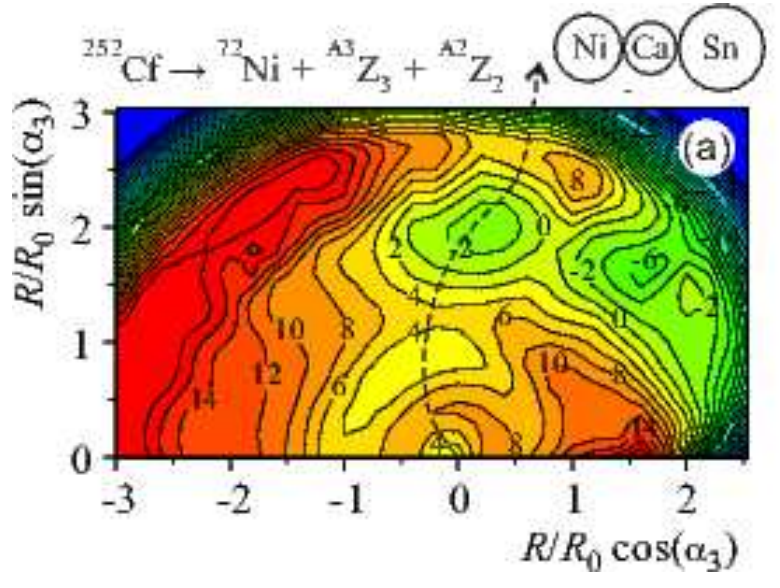

Fig. 11. As in figure 10 the potential energy surface for ternary mass split of ${ }^{252} \mathrm{Cf}$ as function of the elongation of the system and the mass of the third (central) fragment, from Ref. [28]. This figure compared to the previous figure shows the influence of the shell effects, which distinctly change the landscape. Results obtained with the three-center shell model, with the shell effects included. The dominant decay is with ${ }^{132} \mathrm{Sn}$ as the heaviest fragment with the smallest "Ca"-fragment at the center. The case with a closed-shell Ni-isotope is shown. The dashed line shows the ternary fission path with two barriers.

mation of fragments, the further decay (from the scission point) is governed by the phase space. The fission process can proceed towards several mass partitions, their individual phase space will be different according to the peculiarities.

With the PES's we obtain an overview of the different decay channels. The PES's, which are discussed in Ref. 30, are obtained by calculating the interaction between all fragments:

$$
\begin{aligned}
& U\left(R_{13}, R_{23}, Z_{1}, Z_{3}, A_{1}, A_{3}\right)=Q_{g g g}+ \\
& +V_{12}^{(\text {Coul })}\left(Z_{1}, Z_{2}, R_{13}+R_{23}\right) \\
& +V_{13}\left(R_{13}, Z_{1}, Z_{3}, A_{1}, A_{3}\right) \\
& +V_{23}\left(R_{23}, Z_{3}, Z_{2}, A_{3}, A_{2}\right)
\end{aligned}
$$

where $Q_{\mathrm{ggg}}=B_{1}+B_{2}+B_{3}-B_{\mathrm{CN}}$ is the balance of the fragments binding energies in the ternary fission. The values of the binding energies are obtained from the mass tables in Ref. 31; $V_{13}$ and $V_{23}$ are the nucleus-nucleus interaction of the middle cluster " 3 " $\left(A_{3}\right.$ and $\left.Z_{3}\right)$ with the other two, their mass and charge numbers, with the left "1" $\left(A_{1}\right.$ and $\left.Z_{1}\right)$ and right "2" $\left(A_{2}\right.$ and $\left.Z_{2}\right)$ fragments of the ternary system; $V_{12}^{(\mathrm{Coul})}$ is the Coulomb interaction between the two border fragments " 1 " and " 2 ", which are separated by the distance $R_{13}+R_{23}$, where $R_{13}$ and $R_{23}$ are the distances between the middle cluster and two outer clusters placed on the left and right sides, respectively. The interaction potentials $V_{13}$ and $V_{23}$ consist of the Coulomb and nuclear parts:

$$
\begin{aligned}
& V_{3 i}\left(R_{3 i}, Z_{i}, Z_{3}, A_{i}, A_{3}\right)=V_{3 i}^{(\text {Coul })}\left(Z_{i}, Z_{3}, R_{i 3}\right) \\
& +V_{3 i}^{(\text {Nucl })}\left(Z_{i}, A_{i}, Z_{3}, A_{3}, R_{3 i}\right), \quad \text { where } i=1,2 .
\end{aligned}
$$


$A_{1}$

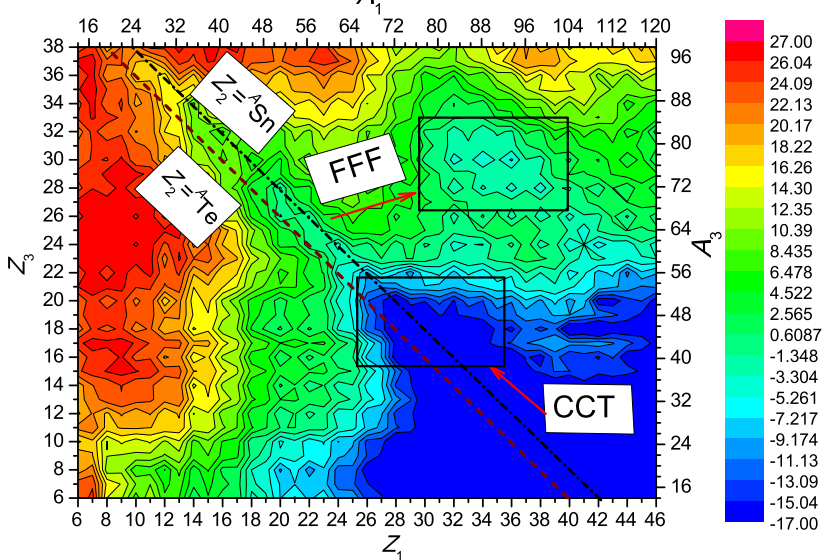

Fig. 12. The contour plot of the potential energy surface (PES) it gives an overview of the different modes for the collinear ternary decays characterized by two values of $Z_{3}$ and $Z_{1}$ of the ternary fragments for ${ }^{252} \mathrm{Cf}$. The pre-scission configurations of the ternary system appear with the isotopes of tin, ${ }^{132-128} \mathrm{Sn}, Z_{1}=50$, and Tellurium ${ }^{134-138} \mathrm{Te}, Z_{1}=52$. The upper rectangle shows the area of the expected mass (charge) distribution of the symmetric ternary FFF-decay products with almost equal fragment masses $A_{1} \approx A_{2}$ and $A_{3} \approx A_{2}$. The lower rectangle $Z_{3}=16-20$ corresponds to the expected region for ternary decays of the two products with the charge numbers $Z_{1}=28-34$ and mass numbers $A_{1}=72-88$ of the ternary configurations leading to the collinear cluster decays (CCT) observed in Refs. 32,33 .

The nuclear interaction is calculated by the double folding procedure with the effective nucleon-nucleon forces depending on the nucleon density distribution (see Ref. [30]). The Coulomb interaction is determined by the Wong formula 34, which also allows us to take into account the deformed shape of fragments and the possibility to consider interactions under different angles of their axial symmetry axis.

\section{Potential energy surface}

We inspect the PES's for the two cases of ${ }^{252} \mathrm{Cf}(\mathrm{sf})$ and ${ }^{236} \mathrm{U}(\mathrm{n}, \mathrm{f})$ in two figures, Fig. 12 and Fig. 13 respectively. We note the "lower" (blue) regions connected to the combinations with the Sn-fragments. Apart from the already known CCT and FFF - decays we observe a pronounced region with a minimum for $A_{3}$, with $Z_{3}=18$, this must be combined as shown in the PES, with two fragments with $Z_{1}=40(\mathrm{Zr})$, or the adjacent value of $Z_{1}=42$, combined with $Z_{1}=38(\mathrm{Sr})$. The particular structure of the isotopes with $Z=18$, have been observed in Refs. 35, 36, they correspond to the new "shells" observed in neutronrich isotopes, as in ${ }^{44-46} \mathrm{Ar}$ (but also in ${ }^{26} \mathrm{Ne}$ ). The same depletion in the PES's is observed in the PES for the case of Uranium fission as shown in Fig. 13. These effects on the masses, which are seen here, are already contained in the recent mass table in Ref. [31. The decays corresponding to these structures are actually contained in the data

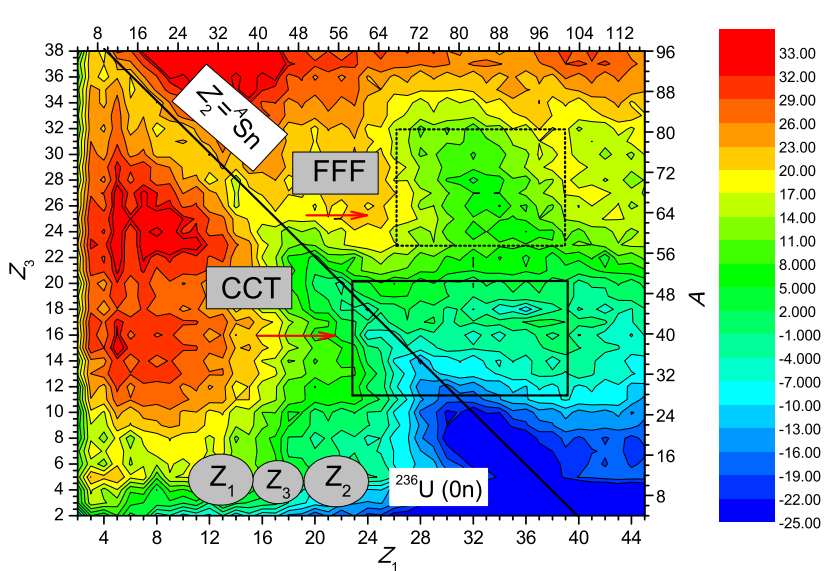

Fig. 13. The potential energy surface for the ternary decays of ${ }^{235} \mathrm{U}(\mathrm{n}, \mathrm{f})$. The general features are similar to the $Z_{3}-Z_{1}$ pattern for ${ }^{252} \mathrm{Cf}$, with a valley dominated by Sn-fragments, $Z_{2}$ $=50$. However, we observe the absence of pronounced effects due $Z_{3}=20,28$ (as in the case of ${ }^{252} \mathrm{Cf}$ ), because for the case of $U$ these fragments are absent. Similarly a region with a lower (green) valley for symmetric decays with comparable three fragments with $Z=32+28+32$ can be favoured (total charge of $\mathrm{U}=92$ ). Again the ternary decays for $Z=18$ (as for the case of ${ }^{252} \mathrm{Cf}$ ) can be favoured, where a proton shell in the fragment makes favorable $Q$-values. These decays are discussed as multi-modal fissions later.

and can be extracted as discussed in the chapter on multimodal fission (in sect. 4.3.

With these figures we illustrate the role of the PES in the formation of the ternary fragments, they point to the differences of the two cases. Clearly the charges have the strongest influence on the phase space in the decays. The effect of the closed shells for protons $\left(Z_{1,2,3}=20,28\right.$ and $50)$ is clearly visible with the low (deep blue) valleys in the PES's. The case of the almost symmetric decays with charges of $Z_{1,2,3}=32,34,32$ is seen as an depletion and points to the possible symmetric decays, these have been discussed in Refs. 37, 38, and are later illustrated in the chapter on multimodal fission in the present survey.

Quite remarkable is the structure of the PES shown in Fig. 14 for the ternary decay of the super-heavy nucleus ${ }^{298} \mathrm{Fl}$, a neutron rich isotope of the recently (Ref. 39]) observed element with $Z=114$. However, with a much larger neutron-number, then observed. We observe the change of the favoured decays with the change of the total charge, and connected to this the influence of the variation of the closed shells in the three fragments.

It is interesting to analyse the situation for the ternary fission for even heavier systems. In heavy systems shell effects in the fragments are not dominant any more, the decay is determined by the liquid drop energies. However, in the fragments the shell with the $Z=50$ remain to be present. Further consequences concerning ternary decays can be deduced from the study of the PES's of very heavy systems like in Refs. 40, 41. In the first case the maximal heavy system is studied, which will have no barrier 


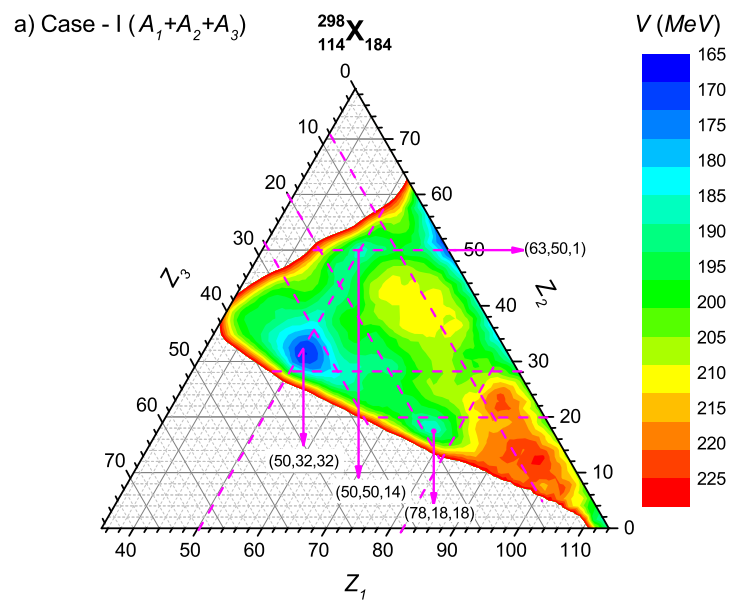

Fig. 14. The potential energy surface for the spontaneous fission decay of a superheavy nucleus ${ }^{298} \mathrm{Fl}$, which has been calculated by Balasubramanian et al. Ref. 40. With the increase of the total charge one region dominates, there are no closed shells (except for $\mathrm{Z}=50$ ) in the fragments.

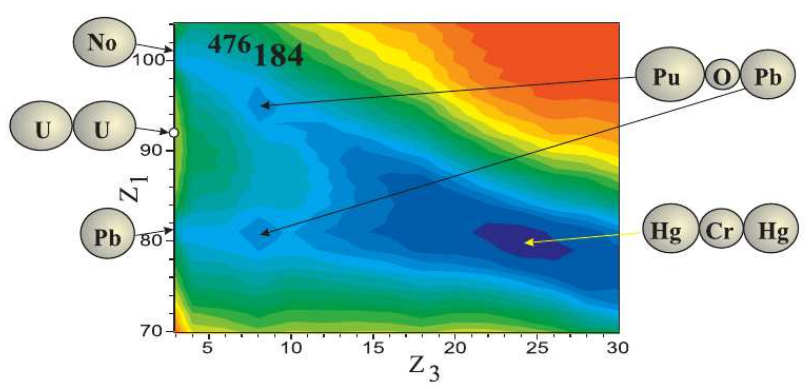

Fig. 15. The potential energy surface for the three-body configurations formed in a collision of two U-nuclei: ${ }^{238} \mathrm{U}+{ }^{238} \mathrm{U}$, which has been calculated by Zagrebaev et al. Ref. 41. With the increase of the tree-body clusterizations become more probable, the shell effect of $Z=82$ is still visible.

for binary fission. In the reaction path of very heavy systems, like for ${ }^{238} \mathrm{U}+{ }^{238} \mathrm{U}$ in Ref. 41, only a few exit channels appear. A binary channel with ${ }^{276} \mathrm{No}_{102}$ and a $\mathrm{Pb}$-isotope could be observed. Further decay channels are shown in Fig. 15. where the tree-body clusterizations with the formation of neutron rich O-isotopes and some heavy clusters $\left({ }^{240-246} \mathrm{Pu}+{ }^{212} \mathrm{~Pb}+{ }^{18-22} \mathrm{O}\right)$ are shown. Looking into these cases it becomes evident, that in the studies of ternary fission the case of Cf-isotopes (and possibly other heavy nuclei/isotopes in the vicinity), which we will discuss, is unique. Many aspects of nuclear structure connected to shells in the fragments and to deformed shells in the total system are present in this case. More cases are discussed by Balasubramanian et al. in Ref. [40], where the ternary decay of heavy nuclei and for a variety of superheavy elements have been studied. In this case shells in the neutron number of the super-heavy elements can be important for the ternary decay.
In very heavy systems, i.e. in reactions of very heavy ions leading to binary and ternary reaction channels, collinear decays have been reported. The dominance of the Coulomb interaction leads to aligned (collinear) ternary fragmentations in higher energy collisions of heavy ions, in particular in deep inelastic collisions in the selection of central impact parameters as described for ${ }^{197} \mathrm{Au}+{ }^{197} \mathrm{Au}$ collisions in Ref. 42 .

\subsection{Sequential decay, shell effects, hyper-deformation, Collinearity}

As already mentioned a three-body decay can be considered to proceed sequentially, each step being determined by the phase space and $Q$-values of each step. From considerations of the PES's the collinear decays are predicted, actually the experimental results have been interpreted as collinear cluster tripartion CCT). This claim from the experimental observations has been contested repeatedly. Most theoretical arguments are based on the strong deep valleys in the potential energy surfaces, which give a favoured phase space for the collinear decays. The collinear decays are actually suggested by the hyper-deformed shapes observed in heavy nuclei. This has been studied experimentally in Ref. [21, for the case of ${ }^{236} \mathrm{U}$ and shown in Fig. 3. These observations favor the collinear geometry relative to "oblate" shapes. In the collinear tripartition of the hyper-deformed nuclear state one of the deformed fragments in the first binary fission starts the sequential decay defining the axial symmetry axis, the following fission process preserves this axis, thus both fission axes are parallel.

These strongly deformed, hyper-deformed, configurations are favored because of the binding energy including shell effects already mentioned, which depend non-linearly on the degree of elongation (shape deformation). This phenomenon has been introduced by Strutinsky 8 and studied experimentally extensively 40 years ago and described in Refs. [18, 19,20]. These deformed shells are quite pronounced at larger values of the quadrupole deformation. The hyper-deformed shapes (axis ratios of 3:1), appear in most of the heavier nuclei due to the deformed shell effects in the total system, and give rise to the observation of fission isomers [16, 17, 18. For spontaneous fission and fission induced by gamma-rays, neutrons or charged particles, the nucleus on its way to fission undergoes extreme deformations with a stretching due the Coulomb forces and inevitably passes through super-deformed and hyper-deformed shapes before splitting. The shell effects in nuclei will delay fission due to the structure with one and two barriers in the collective potential.

In the recent theoretical analysis of ternary fission by Karpov 28, the shell effect discussed previously by Strutinsky [8], suggests that deformed shells are most important for the ternary decays, as well for the binary fissions. In this work 28], based on the three-center shell model, the shell structures in all fragments give rise to a complex deformation of the total fissioning system, which is equivalent to hyper-deformation. The potential energies for the 


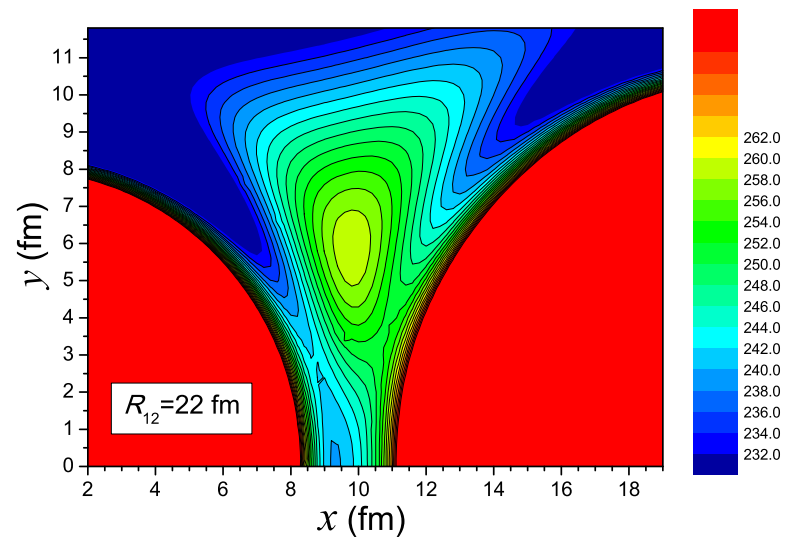

Fig. 16. The potential energy surface $V\left(R_{12}, x_{3}, y_{3}\right)$ as a function of the position $x_{3}$ of the middle fragment " 3 " (Ca) along axis $R_{12}$ connecting the centres of the outer fragments " 1 " and "2" of the tri-nuclear system and $y_{3}$, which is perpendicular to $R_{12}$ at the value of $R_{12}=22 \mathrm{fm}$. The minimum value of the potential well for Ca is $V_{\min }\left(R_{12}=22 \mathrm{fm}, x_{3}=9.4 \mathrm{fm}, y_{3}=0\right.$ $\mathrm{fm})=239.75 \mathrm{MeV}$.

fission path have been shown in Figs. 10 and 11. With this work the most complete description for the dynamics of the decay, particularly related to the barriers has been obtained. There still remains the final dynamics of the ternary decay, which will be governed by the phase space, the latter being determined by the potential energy surfaces PES'S (generally obtained with an approach with three deformed clusters).

The ground state shape of the actinide nuclei is prolate [43, the shape is the result of the balance between the repulsive Coulomb and attractive surface tension forces. The prolate shape in the ground state is favorable energetically for the three massive fragments of the collinear ternary fission. Authors of Ref. 44] found that the potential energy of the collinear shape is significantly lower than the one for the oblate shape and the relative yield of the ternary fission products is much larger for the prolate case than for the latter shape.

Actually with the formation of the necks due to deformed shells, quantum mechanical fluctuations are expected, an almost simultaneous (a sequential within a very small time delay) break up of the two necks is expected for the ternary scission as described in Refs. [45, 46]). In these calculations also the more asymmetric binary fission is predicted and finally a three cluster configuration due to the shell structures for protons and neutrons appears in the exit channel. Actually the shell effects give for both, binary and ternary decays a dominance for the decay with fission fragments with mass $A=132(Z=50, N=82)$.

In the work of Tashkhodjaev presented in Refs. 45, 46] an explicit quantum mechanical calculation of the motion of the fragments towards scission and separation has been done. According to results of this calculations in Ref. 47, the nuclear interaction between fragments plays a decisive role in the pre-scission geometry of the tri-nuclear system. The collinear configuration of the tri-nuclear system

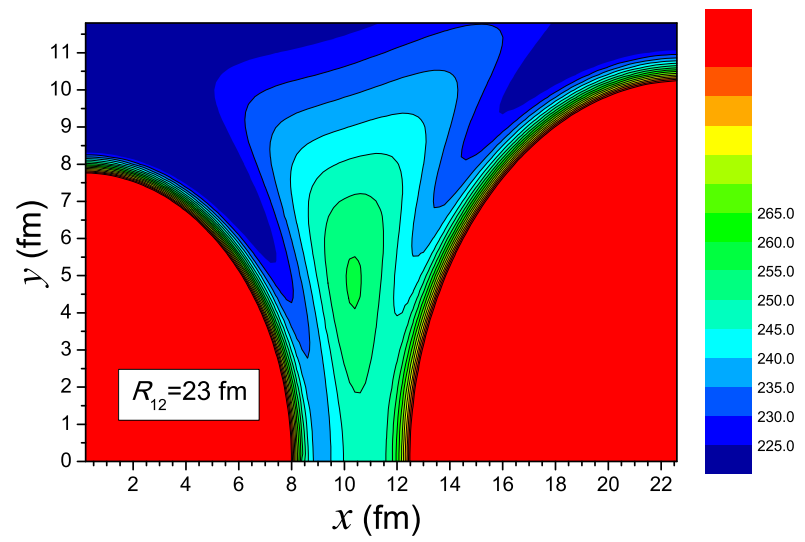

Fig. 17. The same as in Fig. 18 but for the larger distance $R_{12}=23 \mathrm{fm}$. The minimum value of the potential well for $\mathrm{Ca}$ is now $V_{\min }\left(R_{12}=23 \mathrm{fm}, x_{3}=9.0 \mathrm{fm}, y_{3}=2.2 \mathrm{fm}\right)=236.83 \mathrm{MeV}$.

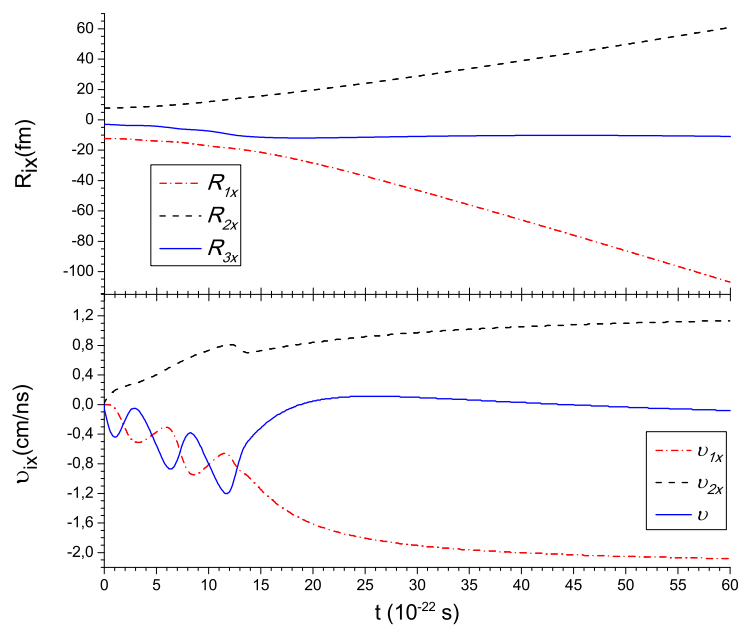

Fig. 18. The $x$ component of the coordinates (upper part) and the velocities (lower part) in the decay into three fragments as functions of time, from Ref. [47.

(TNS) is preferable for the values of the distance between outer nuclei " 1 " and " 2 " being in region of $R_{12}=21-22$ $\mathrm{fm}$, but the fluctuation of the position of the middle $\mathrm{Ca}$ cluster (nucleus " 3 ") from the collinearity axis can be observed due to the extension of the potential well in the interaction potential between the fragments of the TNS up to $2 \mathrm{fm}$ around the axis connecting the centres of the outer nuclei (see Fig. 16). At the distance $R_{12}>22 \mathrm{fm}$ the overlaps of the nucleon densities of the TNS nuclei decrease and, as a result of the decrease in the nuclear attraction and the Coulomb repulsion the depth of the potential well decreases. In that way the condition for the separation of the middle fragment $\mathrm{Ca}$ from $\mathrm{Ni}$ can arise. As a result the potential well for Ca-fragments moves away (perpendicular) from the $x$-axis at values of $R_{12}=23 \mathrm{fm}$ and its minimum value decreases: $V_{\min }\left(R_{12}=23 \mathrm{fm}, x_{3}=9.0 \mathrm{fm}\right.$, $y_{3}=2.2 \mathrm{fm}$ ) $=236.83 \mathrm{MeV}$ (see Fig. 17). 


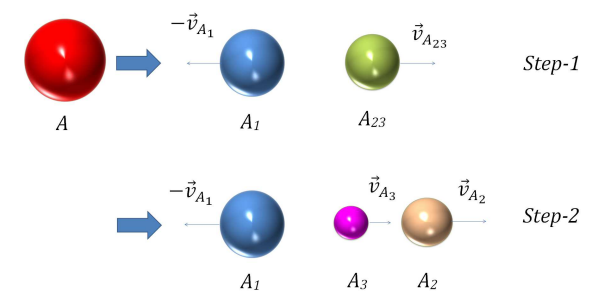

Fig. 19. Illustration of the model of a sequential decay for the collinear ternary fission proceeding in two steps. In the first step the combination $A_{23}+$ fragment $A_{1}$ are formed, followed (within a very short time sequence, in $10^{-21}$ seconds) by the fission of $A_{23}$ into $A_{2}$ and $A_{3}$.

The local minimum at point $R_{x_{3}}=9.4 \mathrm{fm}$ and $R_{3 y}=0$ fm (see Fig. 17) moves to the left (to the side of the $\mathrm{Ni}$ nucleus), and starting from $R_{12}=23 \mathrm{fm}$ this minimum point is transferred to a saddle point. In Ref. [46] the authors have considered the case when initially all nuclei are placed in one line, which means that $R_{y_{1}}(t=0)=R_{y_{2}}(t=$ $0)=R_{y_{3}}(t=0)=0$, since the energy of the collinear configuration in the pre-scission state is the smallest, and the values of the $x$-coordinates of these nuclei (with the smaller relative distance between nuclei) correspond to the local minimum in the Fig. 17. Both components $(x$ and $y$ ) of the initial velocities of the three nuclei are zero. In other words the formation of fragments of the TNS is so slow that the fragments have zero (or very small) velocities. Nevertheless, the assumption that all initial velocities are zero, means that there is no net force, which acts on the nuclei in the equilibrium state. Results of the calculations of the equations of motion with the above mentioned initial conditions are shown in the Fig. 18. There it is shown that from the beginning, the Sn nucleus is going to breakup into the $\mathrm{Ni}+\mathrm{Ca}$ system, and then at a time of $t \approx 13.5 \times 10^{-22} \mathrm{~s}$ the $\mathrm{Ni}+\mathrm{Ca}$ system has decayed. Moreover, an important result has been obtained, namely that the central third nucleus $(\mathrm{Ca})$ has almost not changed it's coordinate, because it's velocity is about zero. This means, that the detection of the middle nucleus (Ca) is almost impossible in an experiment. All together these conditions lead to collinear fission of the tri-nuclear system. In fact in a similar recent study by Vijayaraghavan et al. 48 the trajectories of fragments in a ternary decay have been calculated explicitly for equatorial and collinear trajectories. For lighter fragments an emission at relative angles of $90^{\circ}$ is obtained. For third fragments of intermediate mass for an initial configuration with the fragment on the axis results in a collinear decay.

As a further consideration we look into the kinetic energies of the three fragments with a model of a sequential break up of the fissioning nucleus (illustrated in Fig. 19.

The collinear decay mode is quite important in the analysis of features of the fission fragments, in particular their kinetic energies. Several authors have addressed this point [22,49]. In Ref. [49] an overall study of the probabilities of ternary decays is given, comparing oblate and prolate fission. Ternary decays become dominant for cases where the central mass becomes larger than $A_{3}=30-40$. Considering the potential energy as function of the size of the central (smaller) fragment, $A_{3}$, it has been shown that for larger central fragments, the ternary mass splits dominate the decays of heavy nuclei by orders of magnitude. Further the dependence of the potential energy for ternary mass splits on the geometry of the ternary system has been analysed by Vijayaraghavan et al 22 for different orientation angles between the three fragments as shown in Fig. 20

In order to put the collinear geometry into the perspective of the potential energy of the system, we can consider the potential between three clusters in dependence on an angle between the axial symmetry axis of a two-fragment subsystem and the third fragment, defining different shapes of the decaying system (Fig. 20 ) In this work of Vijyaraghavan [22] the potential energies of three fragments in different configurations have been calculated. In Fig 21 we show the potential energies of the fragments for the three-cluster system, where the collinear and oblate arrangements are defined by the angles shown in Fig. 20 , by varying the orientation angle for the three clusters. We note the lowest potential energy for the arrangement with $\mathrm{Ca}$ as the central fragment.

The potential energies are clearly lowest for the collinear geometry. Around the lower region of the potential energy quantum mechanical fluctuations around the minimum must be expected. This is an effect of the uncertainty principle discussed in Ref. [52. This has also been considered in Ref. 23, where the question of the angular distribution of the ternary fragments is addressed. The considerations of the macroscopic features using clusters in the ternary nuclear system can, however, only give a general view, which describes mainly the properties of the asymptotic phase space of three fragments reached by the ternary decay.

For the discussion of the experimental results the kinetic energies of the fragments are of importance, they have been calculated in Refs. 23,50. For this study the collinear cluster tripartition is defined to proceed in two steps, in the first step an intermediate fragment defined as $A_{23}$ is formed with some excitation energy, as shown in Fig. 19 , in the second step the fragments $A_{3}$ and $A_{2}$ are formed in a binary decay. The fission barrier of the first step is small in comparison with the barrier of the second step, which is considerably higher then the first one. Note the fission barriers defined here are obtained with the depth of the potential well in the landscape of the potential energy surface, i.e. the value of the barrier relative the bottom of the minimum. The absolute value of the barrier is the Coulomb barrier, which determines the total kinetic energy of the fission products. These kinetic energies have been shown in Fig. 4, as function of the mass of the central fragment, $A_{3}$ and for different excitation energies of the intermediate fragment $A_{23}$. A considerable value for the excitation energy of $A_{23}$ appears for a large fission probability in the second step. Actually a value of at least $30 \mathrm{MeV}$ must be assumed, in this case the kinetic energy of the central fragment $A_{3}$ is close to zero, similar 


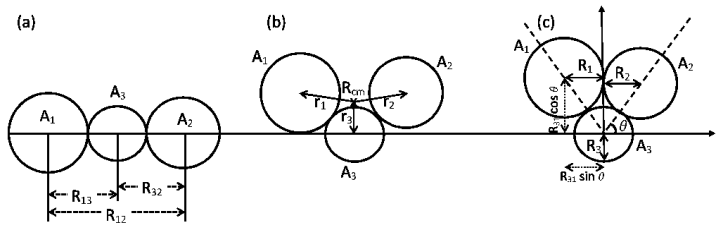

Fig. 20. The coordinates of three clusters defined with an orientation angle of fragment $A_{3}$ relative to the axis between the two other fragments, from Ref. [22]. The corresponding potential energies are shown in Fig 21.

to the other approaches discussed in Ref. [23, 47] and in Ref. [28. The kinetic energy $E_{k i n_{A 2}}$ of $A_{2}$ is maximum and reaches values of $150 \mathrm{MeV}$. This is actually an artefact of the model as pointed out in Ref.[26], because no intrinsic excitation of the three fragments are taken into account. In this reference the energy correlations between the two outer fragments $\left(A_{1}\right.$ and $A_{3}$, obtained with the experimental setup COMETA) show that the kinetic energies show several groups with much lower kinetic energies. Most important for the experimental circumstances is the fact that the central fragment usually is lost due to it's low kinetic energy by being absorbed in the target and/or in the target-backing.

The same result for the kinetic energies has been obtained in the work of Holmwall et al. 23. in explicit calculations of the trajectories. Their approach to calculate the kinetic energies is based on the "Almost sequential decay", as used in the approach of Ref. [47. In this work also the dependence on various parameters of the decay are shown, namely the dependence on the possible neutron emission (neutron-multiplicities), the effect of lateral momenta and the variations in initial geometrical positions of the central clusters. For deviations from the central position, it is concluded that they destroy the collinearity in the final channel. Actually a detailed analysis of the kinetic energies of the fragments and the dynamical constraints for the decay as done in Ref. 23 within a model with existing clusters (as in many other approaches) gives results, which prevent the ternary decay, in contradiction to the experimental observations. The authors of Ref. [23] did not include the nuclear interaction in the calculation of the nucleus-nucleus potential. As a result the kinetic energy of the outgoing fragments had been overestimated. As a conclusion we can state that these approaches based on preformed clusters without deformation of the fragments, fail to describe the experimental results.

More relevant are the concepts with a continuous deformation path like on hyper-deformation, discussed before and in the approach of Karpov 28, where the total system with three nuclear centers is used all along the fission path. In the latter approach also two barriers exist, which are strongly influenced by the shells, in particular the second barrier (which is higher) is very strongly reduced by the shell effects in the fragments.

Initially ternary fission has been defined by the observation of a third light particle emitted perpendicular to

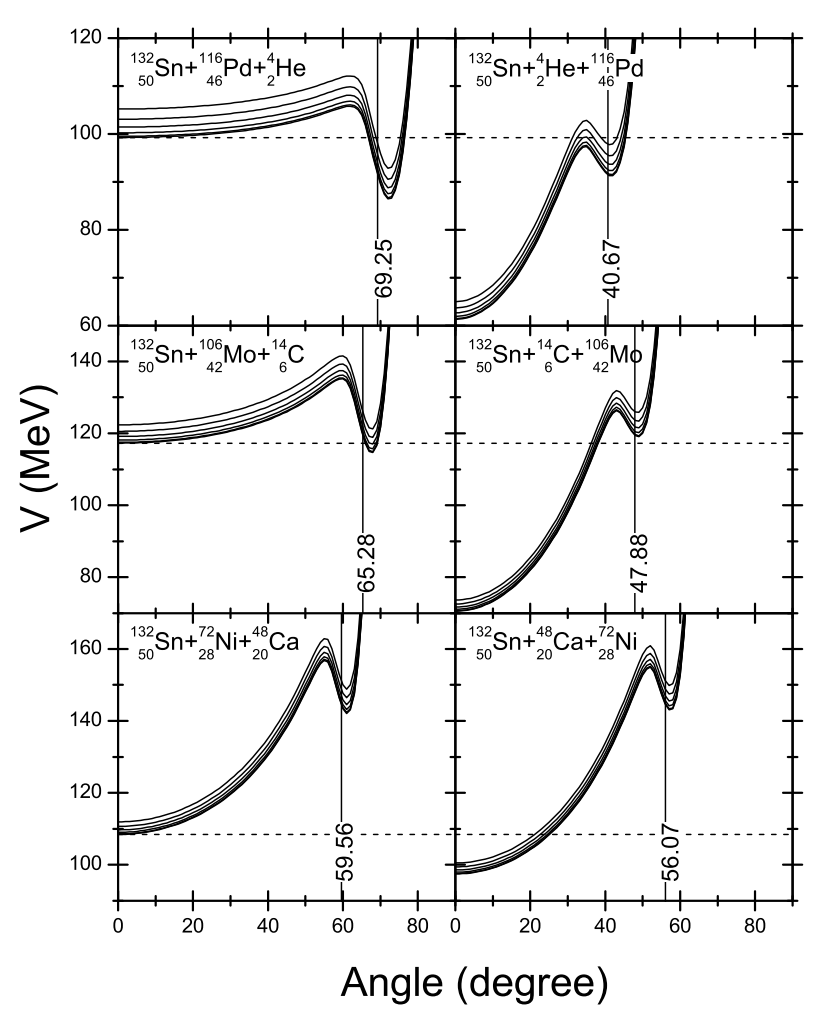

Fig. 21. The potential energies of three clusters defined for different orientation angles for the three clusters for the combination $A_{23}+A_{1}$ as defined in Fig 20. Three cases for the central particle are considered, with ${ }^{4} \mathrm{He},{ }^{14} \mathrm{C}$ and ${ }^{48} \mathrm{Ca}$

the fission axis in coincidence with the binary fission fragments. The most important feature, which appeared with the study of true ternary fission is the fact that the decay into three comparable mass fragments becomes collinear. In the experiment by Schall et al. [51, the question of the decay from oblate deformed shapes is addressed, typically a decay with three similar vectors was expected. The experiment has been designed to detect ternary fission events with the emission of three heavier fragments at relative angles of $120^{\circ}$. The experiment covered a large solid angle using several ionisation chambers (detectors) each covering a large angular range of $\left(90^{\circ}\right)$ degrees, designed to observe over a large solid angle the triangular shape of the decay-vectors. This experiment gave a negative result, with an upper limit of the probability for this decay of $1.0 \cdot 10^{-8} /$ (binary fission). The failure in observation of true ternary fission in this experiment is explained by the very small probability of the population of the configuration which is presented in Fig. 20(c). The potential energy barrier of the last configuration is very high and its population needs higher excitation energy for the fission to occur. This argument is illustrated in Fig. 21 where the potential energies of three clusters are presented for different orientation angles between lines connecting the centres of mass of the three clusters. 


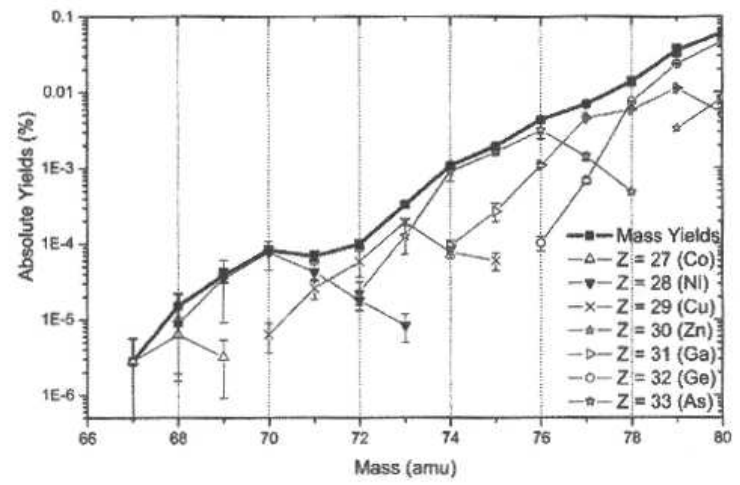

Fig. 22. Very mass asymmetric fission yields of the ${ }^{245} \mathrm{Cm}(\mathrm{n}, \mathrm{f})$ reaction. (adopted from Ref. 55]). The fission yield around mass $A=70(Z=28)$ is enhanced.

\subsection{Deformed shell effects, symmetric and very asymmetric binary fission}

In fission the interplay of the macroscopic (liquid drop) and microscopic (nucleon orbits) effects are most important [8, 9,53,54]. A triple humped barrier is observed in several cases, giving rise to important phenomena, like fission isomers, multi-modal binary fission and creating the way to ternary fission as illustrated in Fig 3 . Binary fission has an asymmetric distribution of the masses with the isotope ${ }^{132} \mathrm{Sn}$ appearing in almost all fission decays [11.

In the very asymmetric binary fission lighter fragments with masses $A=60-80$ can be observed. In these cases "cold" fragments may appear and and deformed shell effects are important. In several works 55 experimental results on very asymmetric binary fission have been obtained, these are single arm measurements. An example of such experimental results is shown in Fig. 22, In these cases the yields of masses around ${ }^{70} \mathrm{Ni}$ are enhanced 55, in particular for the higher kinetic energies of the fragments. These yields in the region of the lighter fragments of $A=70$, can be due to special shell effects, in particular the deformed shells around ${ }^{70} \mathrm{Ni}(Z=28, N=52)$, or due to ternary fission..

\section{Recent experimental results on ternary cluster decays in ${ }^{236} \mathbf{U}(\mathbf{n}, \mathbf{f f f})$ and ${ }^{252} \mathbf{C f}(\mathbf{s f})$}

Inspecting the recent experimental observations and the numerous theoretical predictions [44,56,57, we can state that in the heavy systems and for ternary fragments with larger charge, ternary collinear decay from a prolate configuration is preferred. We refer to the ternary decays in the present work as to "true ternary fission", see also e.g. Zagrebaev et al. in Refs. [41,57. A more recent survey of clustering effects in binary and ternary fission can be found in the articles by G. Adamian, N. Antonenko and W. Scheid 58. In the work by D. Poenaru and W. Greiner 59 it is shown, that in heavy nuclei collinear ternary decays are observed with increasing probabilities for increasing charge of the total system. For even heavier systems quarterny fission must be considered.

Concerning the experimental evidence for true ternary fission several experiments have been performed at the FLNR in Dubna, to list most of them:

a) with two complete detector telescopes (called FOBOS 32, 60 ) with 180 degrees relative angle for binary coincidences in spontaneous fission of ${ }^{252} \mathrm{Cf}(\mathrm{sf})$ and with the same experimental device

b) neutron induced fission ${ }^{236} \mathrm{U}(\mathrm{n}, \mathrm{fff})$, performed at the reactor in Dubna.

c) Experiments of binary fission for ${ }^{252} \mathrm{Cf}(\mathrm{sf})$ in coincidence with neutrons with a system called "MiniFobos", containing smaller detectors of similar structure as with FOBOS, Ref. 33.

d) Binary coincidences for ${ }^{252} \mathrm{Cf}(\mathrm{sf})$, using PIN-diodes (for energy and timing signals) called COMETA.

The most complete data set has been obtained with the system described in Refs. [32, 33, 60. Quite importantly, supporting the claims made in these works, the multi-modal ternary fission decays predicted from the calculations of the PES's, have been extracted from the complete data in a later stage, see Refs. [37,38.

\subsection{The FOBOS Experiments}

For the observation of the ternary decays with the missing mass method the decay vectors of two fragments, which are identified with all parameters (mass, energy and angle) in a measurement of the two fragments in coincidence is needed. In the rather common single arm experiments one fragment is identified with high precision (mass, energy and angle) with the partner being known under the assumption of a binary decay. However, in the recent experiments, coincidences between two fully identified fragments have been measured with two FOBOS-detectortelescopes 32,60 placed at $180^{\circ}$ (see the illustration in Fig 23). The FOBOS detectors allowed the measurement of time of flight, energy, energy-loss and angle with high precision with a very large solid angle.

The details of the detector structure are shown in a scheme in Fig 24. In Ref. 32 the experimental evidence for the missing mass effect had been explained. Due to the energy loss and momentum dispersion in the support foils for the low pressure parallel plate counters in the front of the Bragg-Ionisation chambers a particular scheme has been considered. In this scheme the third fragment after dispersion in the foils is blocked by the support structure for the foils of the ionisation chambers. Actually in the recent calculations, cited in this work, it appears that the central (third) fragment, will have very low kinetic energies, thus it most probably gets stuck in the material of the support of the target or already in the target itself. Thus the missing mass effect has also been observed with detector systems consisting of solid state detectors (PINDiodes, COMETA-set-up), with a measurement of masses by the time of flight (TOF) and energy parameters. 


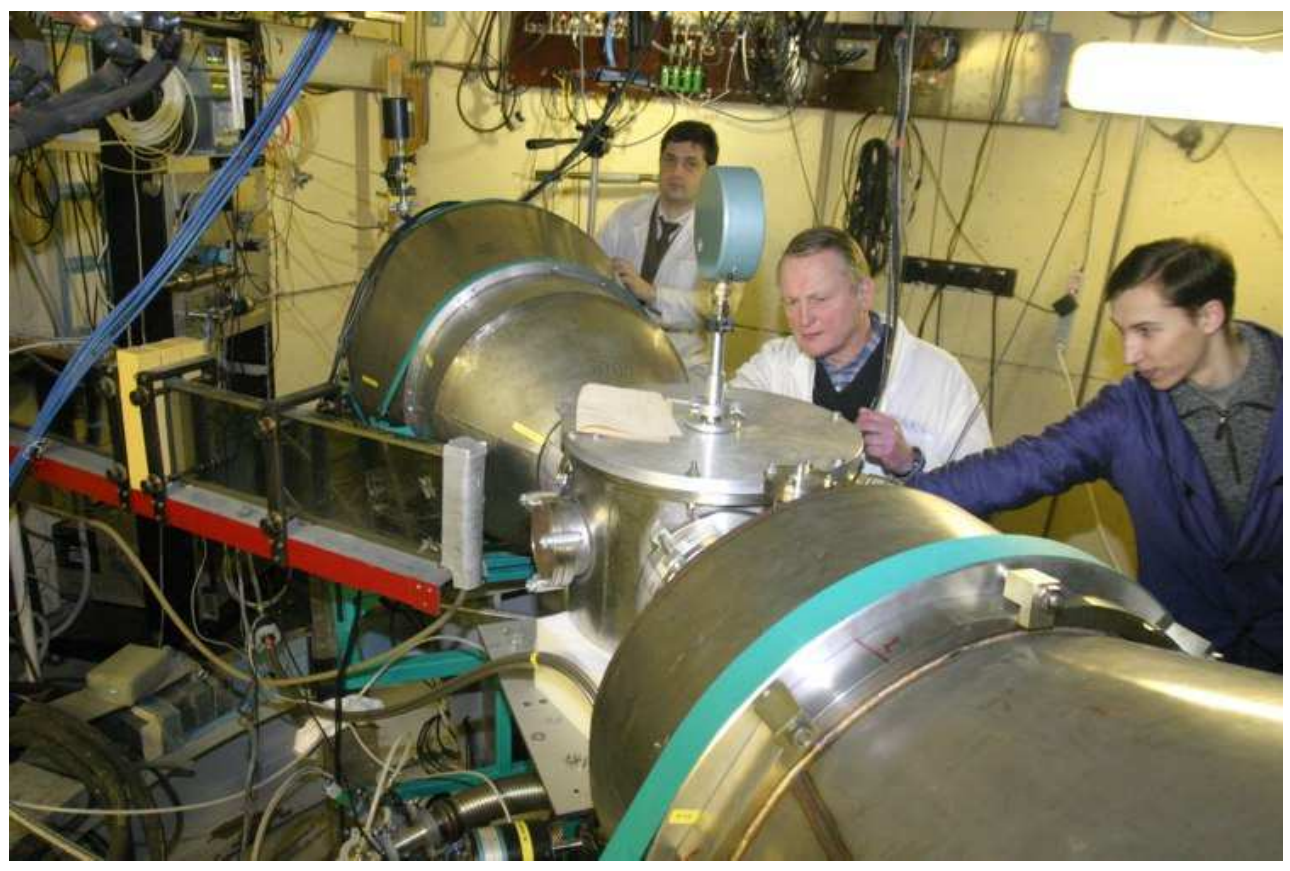

Fig. 23. The two FOBOS detectors placed at a relative angle of $180^{\circ}$ degrees for the measurement of coincidences between two fragments. Two of the leading experimentalists, D. Kamanin and Y. Pyatkov (in the center) are depicted.

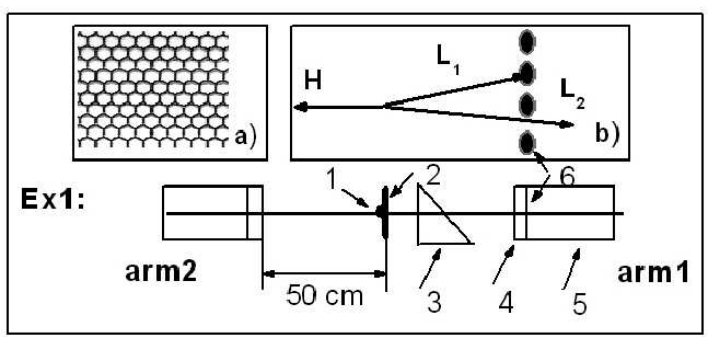

Fig. 24. Scheme of (Ex1) for coincidence measurements of two fragments of the collinear decay of ${ }^{252} \mathrm{Cf}$. Here: $1-\mathrm{Cf}$ source, 2 - source backing, 3 - micro-channel plate (MCP) based timing "start" detector, 4 - position sensitive avalanche counter (PSAC) as "stop" detector, 5 - ionization chamber (BIC) with the supporting mesh, 6 - mesh of the entrance window. The side view of the mesh is shown in the insert (a), a mesh section is presented in the insert (b). After passage of the two fragments through the source backing, two light fragments $\left(L_{1}\right.$ and $L_{2}$, originally fragment $\left.L\right)$, are obtained with a small angle divergence due to multiple scattering. One of the fragments $\left(L_{1}\right)$ can be lost hitting the metal structure of the mesh, while the fragment $L_{2}$ reaches the detectors of the arm1, insert (b). The source backing (2) exists only on one side and causes an angular dispersion in the direction towards the right side (arm1).

In these experiments the binary decay appears as two strong regions of the registered binary fragments in coincidence, as shown in Fig. 26. The determination of all parameters in coincidence with two vectors (angles, mass and energy) gives the complete kinematics for the ternary decay, in these experiments the determination of the missing mass indicated by " 7 " is well determined. This approach has been established, and the phenomenon of collinear cluster tripartition, the CCT-decay, has been described, Refs. 32, 33. The CCT-decay has been observed independently in three different experiments with a missing mass peak: for the spontaneous fission of ${ }^{252} \mathrm{Cf}$ (sf,fff), and in another experiment (at the neutron beam of the nuclear reactor at the JINR) with the same experimental set-up for neutron induced fission in ${ }^{235} \mathrm{U}\left(\mathrm{n}_{\mathrm{th}}\right.$, fff $)$, see Refs. 32, 33. In the latter case with U-nuclei the missing mass turns out to be smaller (as expected), namely it is observed as isotopes of $\mathrm{Si}(Z=14)$, corresponding to smaller charges and masses due to the smaller total mass of the fissioning nucleus, with $A=236$. Further observations, which confirmed these results have been obtained with very different experimental arrangements with the system called COMETA. In the fission mode in the two cases ${ }^{252} \mathrm{Cf}$ (sf,fff) and in ${ }^{235} \mathrm{U}\left(\mathrm{n}_{\mathrm{th}}, \mathrm{fff}\right)$, the decay is dominated by fragments which are strongly bound isotopes (clusters, nuclei with closed shells) of $\mathrm{Sn}, \mathrm{Ni}$, and $\mathrm{Ca}$. The latter, $\mathrm{Ca}$ (or $\mathrm{Si}$ ), as the smallest third particles, if positioned along the line connecting the fragments $\mathrm{Sn}$ and $\mathrm{Ni}$, they correspond to a minimum value of the potential energy. The PES calculated for these cases were compared and illustrated in Fig 12 and in Fig. 13

In the experiments of Refs. 32,33, an important experimental feature appears, which leads to unsymmetric experimental observations in the two detector arms. This 


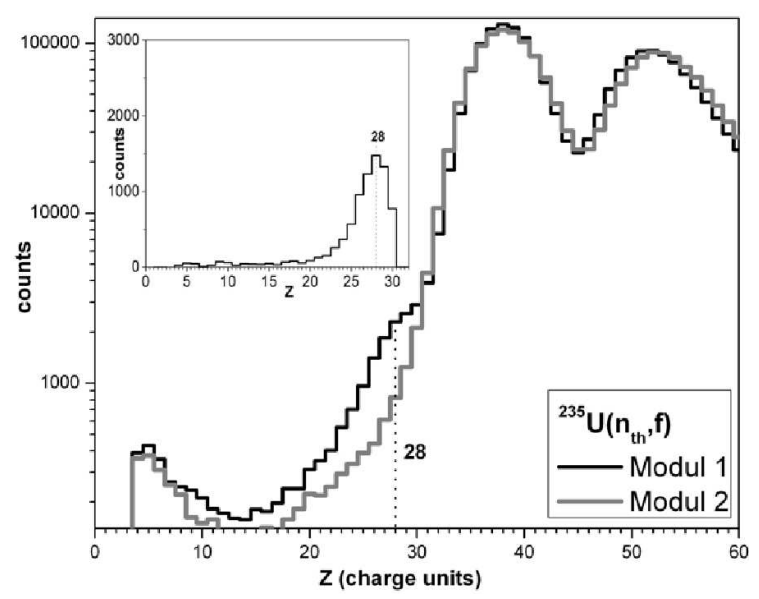

Fig. 25. The energy loss signal derived from the the ionisation chambers of the FOBOS-telescopes. The signals have been extracted from the drift time of the fission fragments tracks in the ionization chambers. Two sides relative to the target are referred to as "Modul 1" and "Modul 2", only one shows the missing fragment yield, according to Fig 26

effect occurs due to the target backing (and the material in front of the detector telescopes) pointing only to one of the detector arms (arm1). Two of the three collinear fragments (2 and 3 ), quasi-bound in the fragment $A_{23}$ move towards one of the detectors (called arm1). Fragments $A_{2}$ and $A_{3}$ are dispersed in angle while passing through the material of the source/target backing and the entrance foils of the detector telescopes. This originally introduced interpretation of the observed effect, can be almost completely relaxed in view of the results (obtained later) on the kinetic energies. These calculations have shown that the central (smaller) fragments attain very small kinetic energy, and they most probably already get lost by absorption in the target and in the target support pointing only into one direction (towards amr1).

With the knowledge on the kinetic energies derived from the more recent calculations (see the kinetic energies in Fig. 40 we can expect that the central fragment $A_{3}$ due to its very low kinetic energy gets lost in all cited experiments. For the alternative case with a small angular dispersion due to the target backing material, it gets dispersed in angle and it is stopped in arm1 on a support-structure (label 6) of the thin foils in front of the Bragg-detectors, see Ref. 32. This fact gives the characteristic side peak in the mass-mass-correlations with a small maximum (label 7) corresponding to a missing mass ( $A=40--48$ ), shown in Fig 26. This is related to the observation the complementary fragments (complementary to $\mathrm{Ca}$ ) the Ni-isotopes in the missing mass effect.

In addition, the signals for the energy-loss have been obtained from the measurement of the drift time of the fission track in the ionization chamber. During the measurement of the time-of-flight of the fission fragments and their energies, two more parameters being sensitive to the nuclear charge are added. The drift time of a track formed after stopping of a fragment in the gas volume of the Bragg ionization chambers is known to be linked to the fragments nuclear charge. Special calibration procedures have been worked out for the nuclear-charge determination of the fission fragments 61. The charge resolution is approximately 3.8 units (FWHM) for the fission fragments in the light-mass peak, the mean values for each charge are correctly determined. The spectrum shown in Fig. 25 shows a clear peak with the nuclear charge of the Ni-isotopes next to the signals of the two charges due to the two fragments of binary fission. At this point we emphasize, that the distributions (the bumps) in mass of all three fragments correspond to a wider range in mass (as opposed to the assumptions in the model calculations in the examples discussed before in this work.

For the neutron induced fission of ${ }^{235} \mathrm{U}$ (n,fff) as shown in Fig. 27 the same features are observed as before, for Fig. 26. however, with a smaller (charge $Z=14$ ) missing mass of 28 - 36 due to the smaller total mass, as described in Refs. 32, 33. The side peak in this case corresponds to some isotopes of Silicon. Therefore the missing mass peak appears closer to the sum of the masses of the binary fragments (sum of the binary masses is now 236). Due to the support (metallic) grid in front of the thin foils in front of the Bragg-ionisation chambers, scattered fragments with high intensity appear in both cases, they form the background shown in Figs. 26 and Fig. 27. This scattered background can be taken from the other detector arm, where this missing mass effect is absent, it defines the background with high counting rates. The final signal is shown in the figures by subtraction of the counting rates in the two arms. Due to the high counting rate in these experiments, the background subtraction can be done with very good precision. We repeat the fact that the two detector arms give different yields, due to the orientation of the target-backing to one of the detector arms, which causes the important difference in the spectra observed, with respect to the detector which "sees the pure target side". The yield in the bump is now $5.1 \times 10^{-3} /$ (binary fission) and it corresponds to the sum all isotopes in the $A_{3}$ fragments occuring in the decay.

\subsection{Fission studies with neutron coincidences}

Further studies of fission decays with coincidences of heavy fragments at relative angles of $180^{\circ}$ and with neutrons detected in coincidence have been performed as described in Ref. 33. The experimental arrangement is shown in Fig. 28. The neutrons are registered in neutron detectors with an assembly, which surrounds the center, where the source for ${ }^{252} \mathrm{Cf}(\mathrm{ff}, \mathrm{fff})$ is placed. For this purpose a modified smaller FOBOS system has been used, shown in Fig. 28, which allows the observation of binary coincidences in coincidence with neutrons.

Fig. 29 shows the mass-mass correlation for ${ }^{252} \mathrm{Cf}$ fissions in coincidence with neutrons. We notice the absence of the "tail" of scattered binary fragments seen in the other cases (Figs 26 and 27). We compare in the lower 

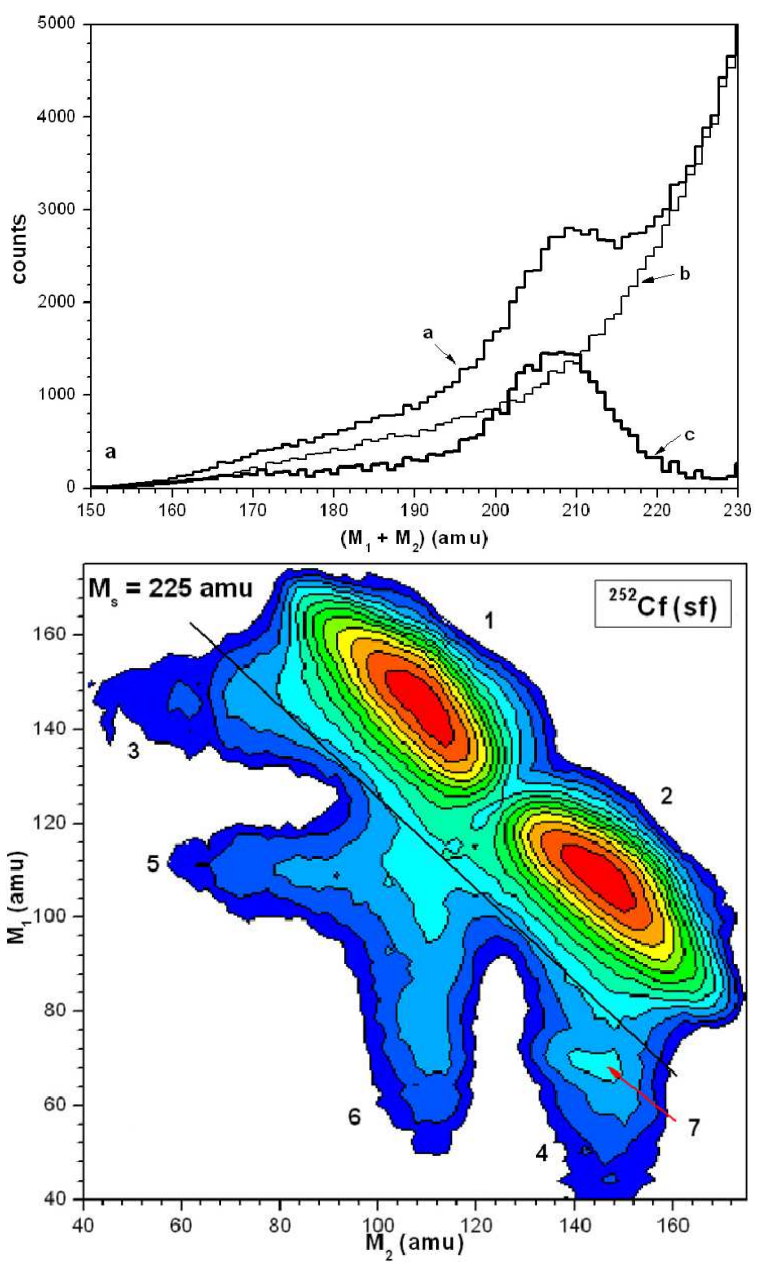

Fig. 26. Upper part: the projection of the mass spectra for the detectors in $\operatorname{arm} 1(\mathrm{a})$ and $\operatorname{arm} 2(\mathrm{~b})$ for spontaneous fission in ${ }^{252} \mathrm{Cf}$. The missing mass spectrum (c) is obtained by the difference. Lower part: shows the original raw data, the coincidences in the two FOBOS-detectors, the contour map of the mass-mass distribution (in logarithmic scale the steps between the lines are a factor 2.5) of the fragments of ${ }^{252} \mathrm{Cf}(\mathrm{sf})$, detected in coincidence in the two opposite arms of the FOBOS spectrometer. The specific bump (missing mass) in the yields in arm1 is indicated by an arrow, 7 . The sum line with $M_{s}=$ $225 \mathrm{amu}$ is shown as an illustration.

part the projections on the mass scales denoted as $\left(M_{1}\right.$ and $M_{2}$ ), the first showing the bumps observed in the Ni-region in the three experiments. For ${ }^{252} \mathrm{Cf}(\mathrm{sf})$, Ex2, denotes the result with neutron coincidences, where no tail of scattered binary fragments appear, for the case of ${ }^{252} \mathrm{Cf}(\mathrm{sf})$ they are visible. The spectra show the sum of masses without the mass of the missing fragment.

Quite remarkable is the observation of the yields in the experimental correlation plot with a definit multiplicity $(n=2)$, shown in Fig. 30 related to decays with missing lighter fragments as central nuclei with the neutron rich isotopes of $\mathrm{Ne}, \mathrm{O}$, and $\mathrm{C}$. In fact, with these lighter frag-

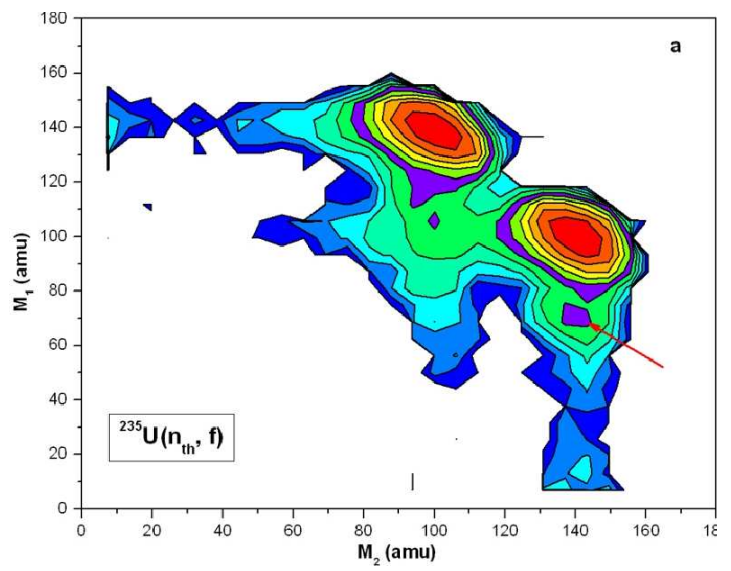

Fig. 27. Mass-mass correlation of the two registered masses measured with the FOBOS detector system for the n-induced fission ${ }^{235} \mathrm{U}(\mathrm{n}, \mathrm{fff})$ at the reactor in DUBNA. The extra missing mass peak indicated by an arrow (compare previous figure) of the ternary decay (due to a smaller missing mass now isotopes of Silicon (Si)) has now a smaller distance to the binary fission fragments compared to the case of Fig 26 , due to the smaller total mass of 236. Projections of the sum of the masses are shown in Fig 29

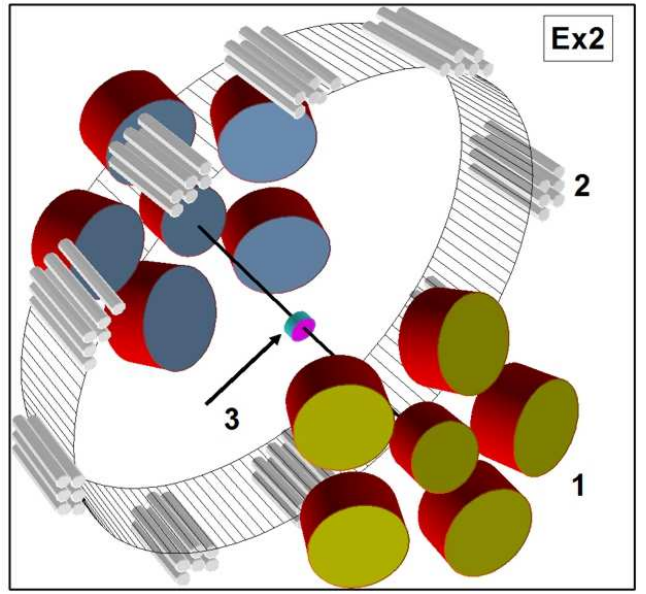

Fig. 28. Experimental Mini-FOBOS set-up for the measurement of correlations of the two fission fragments in coincidence with neutrons. Labels: 1-Minifobos-detectors (Bragg-ionisation chambers), 2-neutron detectors, 3 - source(target).

ments we anticipate an increased neutron emission from the two neck-ruptures, with two heavier fragments at the outside borders of the chains. The projection onto the axis with $M_{1}$ is also shown on the left side. We see an enhanced yield for masses with $A=M_{3}=30$ and $A=M_{3}=70-80$, which indicate the emission of lighter central masses with $Z_{3}=8,10$ as well as for $Z_{3}=20-28$.

In the work in Ref. 33, the neutron detectors have been arranged perpendicular to the fission axis of the bi- 
nary coincidences, defined by the two arrays of MiniFOBOS detectors. This orientation has been chosen to have an increased efficiency for the spontaneous neutrons emitted during fission, potentially from the two necks with the lighter fragments, this explains the observations in Fig. 30

a) Contour map of the mass-mass distribution (logarithmic scale, with lines approximately a step factor of 1.5) from a coincidence in the two opposite arms of Ex2. The bump in the spectrometer arm (arm1) facing the backing of the Cf source is marked by the arrow. b) Projections onto the M1-axis for comparison with the experiment Ex1, and with the results of the $235 \mathrm{U}(\mathrm{nth}, \mathrm{f})$ reaction [1]. Positions of the magic isotopes of $\mathrm{Ni}$ are marked by the arrows (see text of sect. 4.2 for details). c) Projections onto the direction $\mathrm{Ms}=\mathrm{M} 2+\mathrm{M} 1$. The result for Ex1 is presented by two curves marked by the arrows 1 and 2 (dotted) for the arm1 and arm2, respectively. For Ex2 the yield of arm2 is subtracted

With the knowledge from the calculations of the kinetic energies described before (shown in figure 4), we have concluded that $\mathrm{Ni}$-isotopes can be observed as fission fragments with the missing mass of Ca-isotopes in the ternary decay of ${ }^{252} \mathrm{Cf}$. As before in Ex1 and Ex2 the "Ni"-yield can be observed in the COMETA-stup, because the central fragment is absorbed in the source and the source-backing. The experimental result is shown in Fig. 31 and with the projection on the mass scale for the observed Ni-isotopes in Fig. 32. Their overall (summed over the isotopes) yield is equal to $2.5 \times 10^{-4}$ per binary fission. A further discussion of the COMETA-experiment with their results is given in Ref. [26], where energy spectra in correlations between the two heavier fragments $\left(\mathrm{FF}_{1}\right.$ and $\mathrm{FF}_{3}$ ) are discussed. In this work three different energy groups appear, which are interpreted by three different decay scenarios, with $\mathrm{FF}_{2}$ and $\mathrm{FF}_{1}$ in different geometrical arrangements. A big variety of geometrical arrangements of the intermediate system $\left(\mathrm{FF}_{1}+\mathrm{FF}_{2}\right)$ (in the form of pictograms) are proposed, which can produce the different correlated energy groups observed. Most importantly the number of different groups in the energy correlations can be explained, the intermediate system consisting of the fragments $\mathrm{FF}_{1}$ and $\mathrm{FF}_{2}$ gives different energies, in one case the rotation of the intermediate system of $\left(\mathrm{FF}_{1}+\mathrm{FF}_{2}\right)$ is assumed, which gives particular low values of the energies of $\mathrm{FF}_{1}$ and $\mathrm{FF}_{3}$, as observed. Further a critical assessment of other theoretical works is given.

\section{Ternary decays, potential energy surfaces, multi-modal decays}

\subsection{Ternary decays, potential energy surfaces}

Ternary fission into fragments with comparable masses is a process, which occurs in heavy nuclei under conditions of large values of the fissibility parameter: $X$, for the ratios $Z^{2} / A>31$. The decay into three heavier fragments (true ternary fission) is found to be collinear, as observed in the recent experiments and discussed in the previous sections.
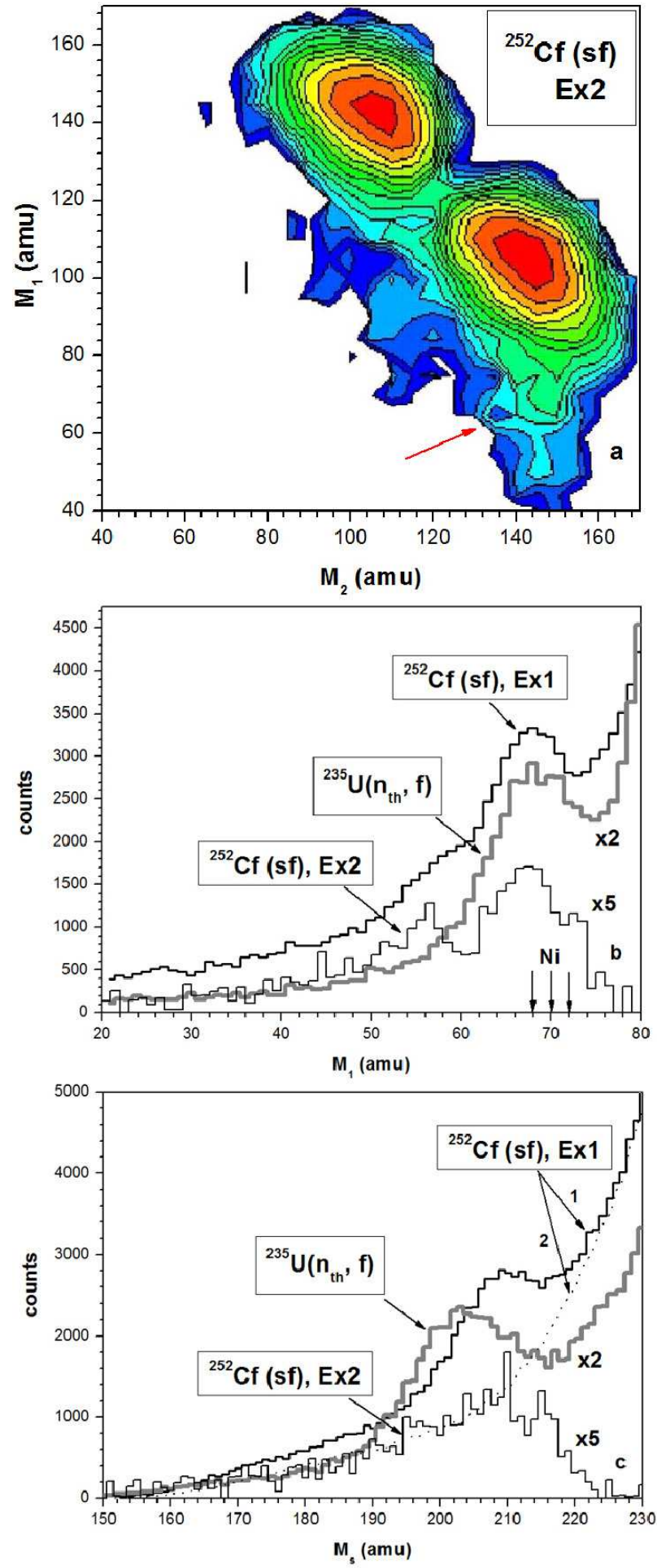

Fig. 29. a) Contour map of the mass-mass correlation of the MINI-Fobos experiment with coincident neutrons. Note the absence of the scattering tails observed in the other cases shown in Fig 26 and Fig 27. Projections (onto the $\mathrm{M}_{1}$ (b) and of the sum, $\mathrm{M}_{s}$, of registered masses (c)) of the yields for comparison with different experiments as indicated; For Ex2 yields of masses from the experiment with neutron coincidences, (MiniFobos) is substracted. 


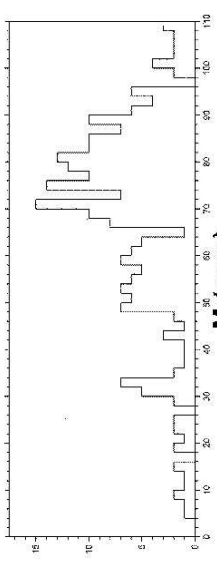

nurez/słunos

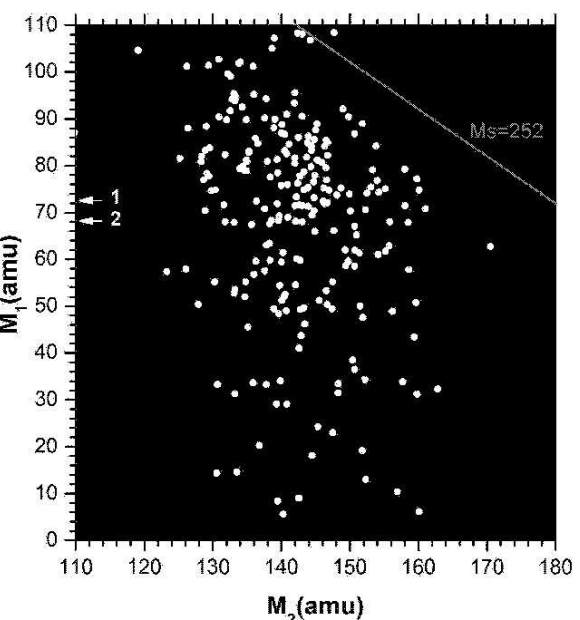

$\mathrm{M}_{2}(\mathrm{amu})$
Fig. 30. The yields of masses from the experiment with a chosen multiplicity in neutron coincidences $(n=2)$, (MiniFobos), shown in a correlation of (M1-M2) and projections on the axis of $M_{1}$ for fragments with missing light fragments $M_{3}$ (with charges Z).

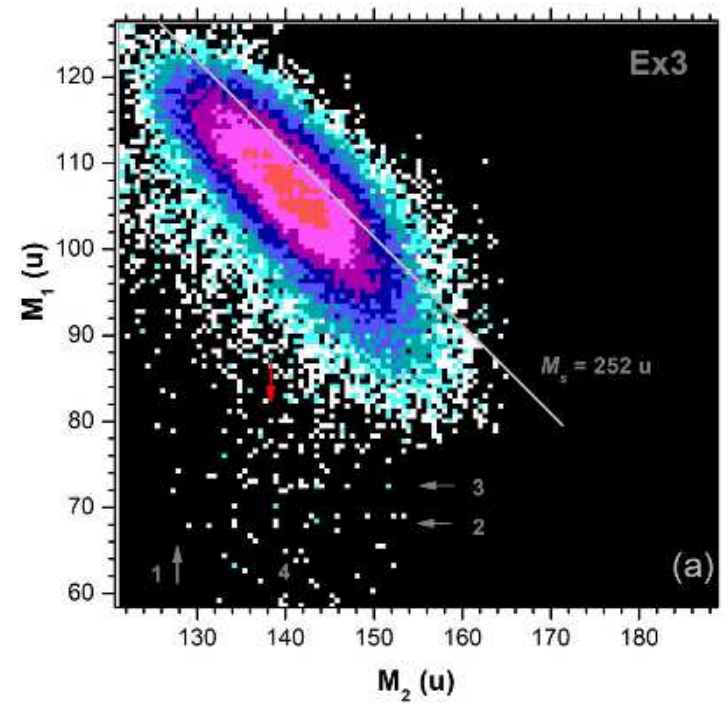

Fig. 31. Binary coincidences of fragments registered with the COMETA set-up. The region of the heavy fragments around masses $A=120-160$ in the arm1 is shown for the dominant binary mass. In addition we observe corresponding to the "Nibump", now Ni isotopes well separated in mass (from Ref. [33]). The Ni-isotopes with mass 68 and 72 (indicated by labels 2 and 3 in the 2D-plot of $\mathrm{M}_{1}-\mathrm{M}_{2}$ ) appear in the projection on the $\mathrm{M}_{1}$ axis, as shown in Fig. 32 . The sum-line $\mathrm{M}_{s}$ of the fragments is shown.

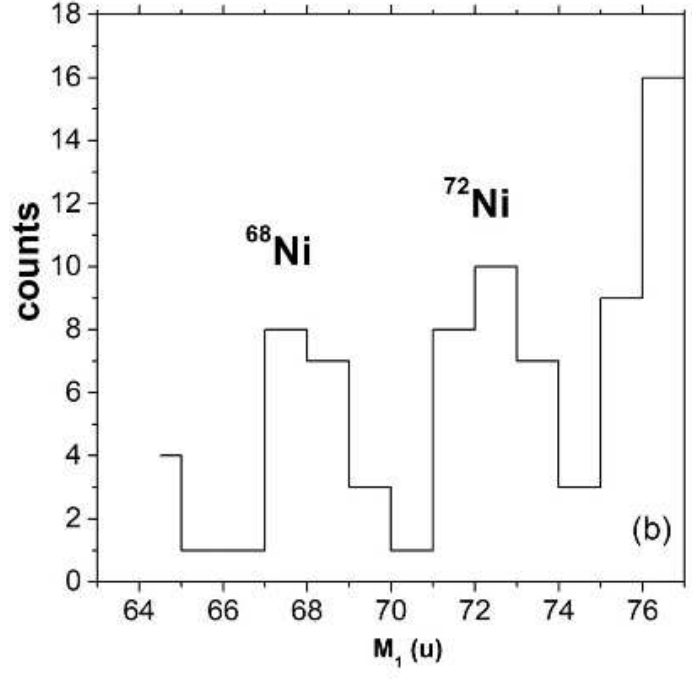

Fig. 32. Projection of the masses observed for the binary coincidences of fragments with masses in the Ni-region (see Fig. 31), registered in the arm1 (from Ref. 33]).

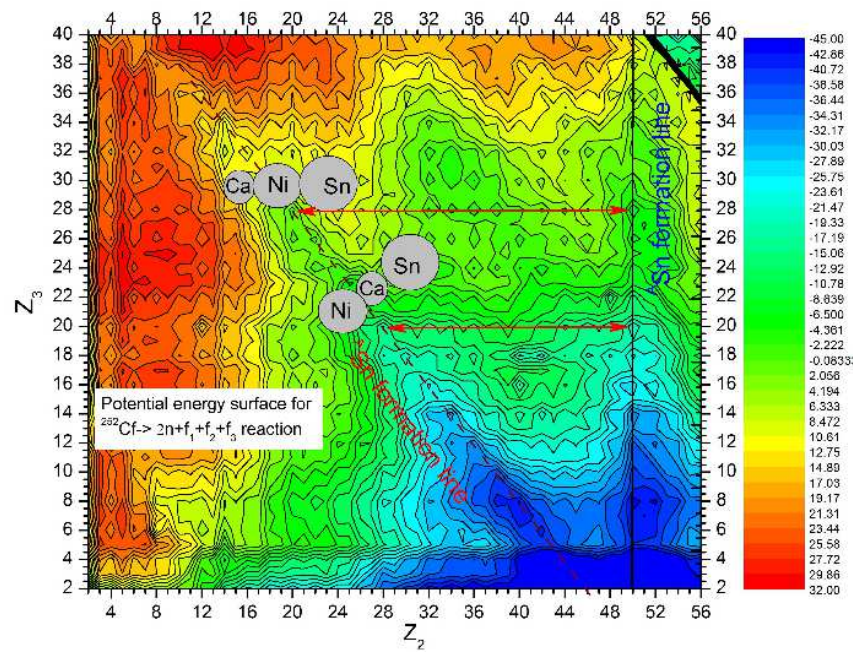

Fig. 33. The potential energy surface for the ternary decay of ${ }^{252} \mathrm{Cf}(2 \mathrm{n}, \mathrm{sf})$, with the emission of two neutrons, fragments $A 3-A 2$ with dominating $A 2$ nuclei with $Z_{2}=50$. The combination of $Z_{2}=50$ and $Z_{3}=20$, or /and $Z_{1}=28$ are clearly seen to be favored.

This important dynamical aspect of true ternary fission has in fact been often predicted in the last decades [14, 44,56]. The ternary decay has $Q$-values which are larger than for binary channels, for the favoured ternary channels this amounts to additional $20-30 \mathrm{MeV}$ see Fig. 7 This fact is observed by inspecting the potentials and the potential energy surfaces for the decay which can be calculated as function of the masses of the fragments formed in the decay.

Binary coincidences are obtained between fragments $A_{2}$, in arm Nr.2 and $A_{1}$ in arm Nr.1 with the missing mass 
method. The masses and their vectors of two fragments are measured, thus the mass $A_{3}$ is uniquely determined, it corresponds to $\mathrm{Ca}$ isotopes in the case of ${ }^{252} \mathrm{Cf}$. This new exotic decay can be understood as the breakup of very prolate deformed elongated hyper-deformed shapes, as discussed for hyper-deformation in ${ }^{236} \mathrm{U}$ in Ref. [21, and shown in Fig 3 . The decay is considered with two sequential neck ruptures [50,62, as illustrated in Fig 19 Actually the central fragment $A_{3}$ has extremely low kinetic energy (see Fig 4) and is mostly lost. The kinetic energies of the fragments have been calculated for the sequential kinematics as shown in Fig.4. We find that the central fragment attains very low kinetic energies, if we assume that the nucleus $A_{23}$ has some intermediate excitation energy of (10-30 MeV).

The main effect in the missing mass FOBOS-experiments with the binary coincidences is the difference in the counting rates (mass spectra) in the two arms of the coincidence arrangement. Two fragments of the ternary decay travel in one, arm1, (see Ref. 32]) through the dispersive media, the source backing and the foils of the start detector. Thus the dispersive effect (angular dispersion of $1-2^{\circ}$ ) of the two fragments from the ternary decay is only present in arm1(!) with the target/source backing pointing to the detector of arm1. Thus the missing mass effect appears in the counting rate difference and the difference in the mass spectra of arm1 and arm2 (the difference $N(\operatorname{arm} 1)$ $N(\operatorname{arm} 2))$. In Fig 26 two mass spectra of arm1 and arm2 and their difference are shown for the case of ${ }^{252} \mathrm{Cf}$, as well as the raw data. The yield derived from the peak in the difference is $4.7 \times 10^{-3}$

For the consideration of the absolute and relative probabilities, the phase space (see below) and the barriers have to be discussed. With the PES for ${ }^{252} \mathrm{Cf}$ we observe several places favored for ternary decays. In order to judge the importance of the various decay channels, the internal decay barriers have been calculated. In the sequential decay mechanism of the ternary fission, the splitting system goes through two corresponding barriers at each step of fission, these barriers are the same for a symmetric decay. These barriers are shown in Fig. 34, where the potential energy of the ternary system ${ }^{70} \mathrm{Ni}+{ }^{50} \mathrm{Ca}+{ }^{132} \mathrm{Sn}$ is shown in a two dimensional plot for the collinear configuration. The motion along $R_{13}\left(R_{23}\right)$ at the fixed value of $R_{23}$ $\left(R_{13}\right)$ is an example for the sequential fission. The case, when $R_{13}$ and $R_{23}$ are increased simultaneously, the simultaneous decay of the ternary system into three parts at the same time will occur. In Fig. 7, the motion along the diagonal line corresponds to the last kind of decay. It is seen from Fig. 34 that the barrier for the simultaneous fission is much higher than the barriers which appear at the sequential fission. This figure allows to judge the relative importance of the decay channels. The symmetric decay with ${ }^{84} \mathrm{Ge}+{ }^{84} \mathrm{Se}+{ }^{84} \mathrm{Ge}$ occurs if the symmetric configuration is populated in the corresponding valley of the potential surface. This figure further shows an important fact: the initially observed CCT-decay with the closed shell nuclei ( $\mathrm{Sn}, \mathrm{Ca}, \mathrm{Ni}$ ) has two different internal barriers, which are the lowest barriers. Variations of the neutron
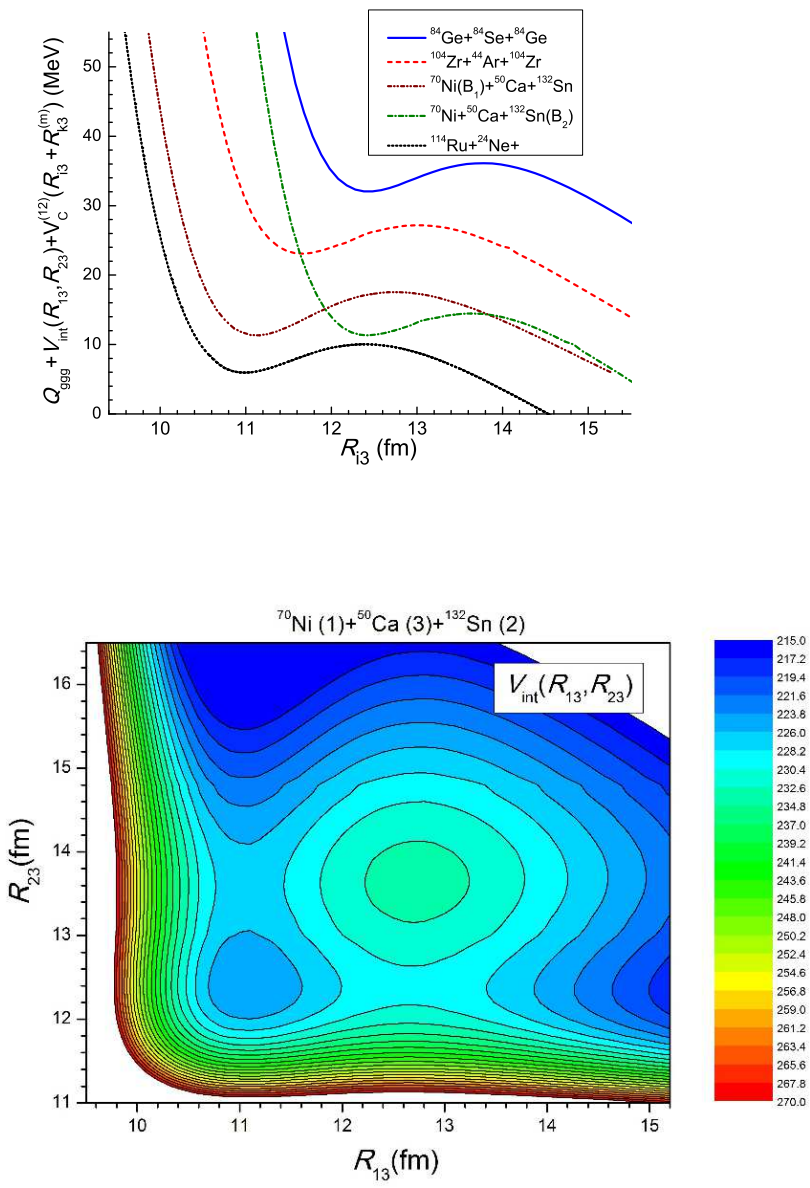

Fig. 34. Upper part: Comparison of the barriers of symmetric ternary collinear cluster decays of ${ }^{252} \mathrm{Cf}$ and other ternary decay modes. The most symmetric decays have the highest barriers. Lower part: the barriers in a $3 \mathrm{D}$ plot for the non symmetric decay into $(\mathrm{Ni}+\mathrm{Ca}+\mathrm{Sn})$, in a correlation of two characteristic distances $R_{13}$ and $R_{23}$.

number adds further favored channels, so the total yield becomes quite large.

The other symmetric channels with ${ }^{44} \mathrm{Ar}$ and ${ }^{24} \mathrm{Ne}$ have lower barriers, we expect that the probability increases for smaller values on the charge and the mass of the central fragment. This hierarchy corresponds to the experimental observations. The barriers of the most important ternary collinear mass splits are shown in the upper panel of Fig. 34. The probabilities for the observation of different modes depend on the formation probability of fragments and on the barriers for the collinear decay channels. For mass-symmetric decays one barrier will be sufficient to characterize the channel, however, for the CCT-decay observed in Ref. 32, 33, with ${ }^{132} \mathrm{Sn}+{ }^{50} \mathrm{Ca}+{ }^{70} \mathrm{Ni}$, fragments as the dominant channel, two barriers are relevant, see the bottom panel of Fig. 34. The experimental observations clearly reflect with their probabilities the influence of the barriers:, the most symmetric mass partition has the lowest yield, the CCT decay is of intermediate proba- 
bility, the CCT decay with a light fragment, like ${ }^{24} \mathrm{Ne}$, in the middle, would have the highest yield $[38,63$.

\subsection{Potential energy surfaces and the multi-modal ternary decays}

The fission processes is a statistical decay of the total (compound) nucleus, CN, as already discussed in 1939 by N. Bohr and J.A. Wheeler 4. Actually the ternary decay occurs with two neck ruptures in a short time-sequence, as discussed in Ref. 30. The probabilities of the ruptures are governed by the internal prescission barriers, by the phase space and by the PES in each configuration. The PES are obtained as described in Refs. 30,64, and are shown in Figs 12 and 13 . The total phase space of these statistical decays is determined by:

i) the energy balance and thus the details of the potential energy surface, PES, namely, its valleys and hills, ii) the internal barriers for the two necks, and iii) the $Q_{g g g^{-}}$ values, the latter determining the kinetic energies and the number of possible fragment (isotope) combinations,

iv) the excitation energy range in the individual fragments, v) their momentum range,

vi) the number of excited states (or the density of states) in each of the fragments, the combinations consisting of 2 (or 3 ) isotopes, and by

vii) the spin $(J)$ multiplicity in these excited states with spins up to $(6-8)^{+}$(phase space factor $\left.(2 J+1)\right)$.

We have seen that the PES for the case of neutron induced fission, ${ }^{235} \mathrm{U}$ (n,fff), shown in Fig 13 , shows different details in structure compared to the ${ }^{252} \mathrm{Cf}(\mathrm{sf})$ case due to the absence of shells in the favored fragments. Details of this decay have been discussed by Tashkhodjaev et al. in Ref. [30, and we show in Fig 35 the yield of fragments obtained in this work. A much wider distribution in masses of fission fragments is observed as compared to the case of the spontaneous fission in ${ }^{252} \mathrm{Cf}(\mathrm{sf})$.

In this work attentions is paid to the fact of the sequential nature of the ternary fission decay, which produces in the first step two fragments with excitation energies, ${ }^{154} \mathrm{Nd}^{*}$ at $25.3 \mathrm{MeV}$ and ${ }^{82} \mathrm{Ge}^{*}$, which leads to the evaporation of neutrons. In the second step the fission of ${ }^{154} \mathrm{Nd}^{*}$ into ${ }^{72} \mathrm{Ni}+{ }^{80} \mathrm{Ge}^{*}$ will occur. These predictions, with a yield of $1.5 \times 10^{-4} /$ (binary fission) are in very good agreement with the experimental observations.

\subsection{Multi-modal ternary decays}

In Fig. 30, we show the yield in an experiment Ref. 33 with coincident neutrons (multiplicity $n=2$ ). This experiment selects a region of lighter missing masses of ternary fragments. These light neutron-rich fragments are typically created at the center of the system with the emission of neutrons. The area is seen in the lower part (blue area) of the PES's (in Figs.8 and 9). The projection on the A1 - axis shows that these are isotopes of Neon, Oxygen and Carbon. The examples indicate, that the multiple modes of the ternary fission decays, can successfully be predicted

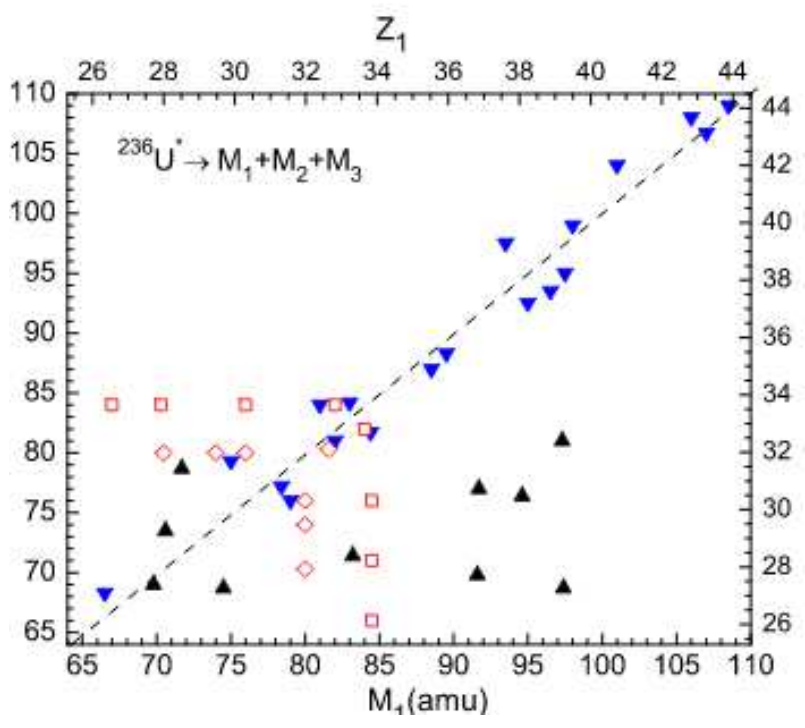

Fig. 35. Results of the calculation by Tashkhodjaev et al., 30, for the fragments yields of sequential ternary fission in ${ }^{235} \mathrm{U}$ (n,fff). The wider (as compared to ${ }^{252} \mathrm{Cf}(\mathrm{sf})$ ) overall spread in masses of fragments is obtained, as predicted from the PES in Fig 13 Up triangles, results of the experimental data selected. Red diamonds and red squares the result of symmetric (sequential, with approximately equal momenta) decays ${ }^{235} \mathrm{U}^{*}$ $->\left({ }^{154} \mathrm{Nd}^{*}+{ }^{82} \mathrm{Ge}^{*}\right)->{ }^{80} \mathrm{Ge}+{ }^{72} \mathrm{Ni}$ and $\left({ }^{150} \mathrm{Ce}^{*}->{ }^{82} \mathrm{Zn}\right.$ $\left.+{ }^{72} \mathrm{Ni}\right)+{ }^{82} \mathrm{Ge}^{*}$. Fragments with comparable masses have been selected.

with the PES's, however, it can be very difficult to extract the corresponding yield from the raw data.

For the ternary decays of ${ }^{252} \mathrm{Cf}$ with a collinear arrangement of the three fragments the PES's, the contourplot in Fig. 27 show distinct minima for various charge combinations (multi-modal ternary decays) with $\Sigma_{Z}=$ 98:

i) for CCT $\underline{Z_{3}}=20$, and $Z_{1}=28$, this is the main CCTmode observed in Refs. 32 and

ii) less pronounced are charge combinations $Z_{3}=28$, and $Z_{1}=20$, as observed in Ref. 32,33. The complementary fragments with $Z_{2}$ are isotopes of $\operatorname{Sn}(Z=50)$. The PES shows a pronounced valley with charge $Z_{2}=50$ and neutron number $N_{2}=126$, due to the closed shell for the number of protons and neutrons. Because of the dominance of the Coulomb interaction, the proton shells are the most important. The individual probabilities vary over several orders of magnitude, see also Ref. [38.

iii) The ternary fissions with $Z_{3}=18$, are predicted.

iv) We observe a pronounced region of minima for the symmetric charge combinations with three comparable fragments, for the FFF-decays (this decay is marked as FFF): for $\left(Z_{1}=32,34,32\right)$, and $\left(Z_{3}=34,32,32\right)$, the fragment $Z_{2}$ has an equivalent role as the other two $Z$-values $Z_{2}$ $=32,32,34$ (since $\left.Z_{1}+Z_{2}+Z_{3}=98\right)$ they can be interchanged - we have an almost symmetric ternary decay. Because of this fact, permutations of the labels including $Z_{2}$ in the figure of the PES (see also Fig 7) will produce similar results, and a symmetric shape of the coincident 


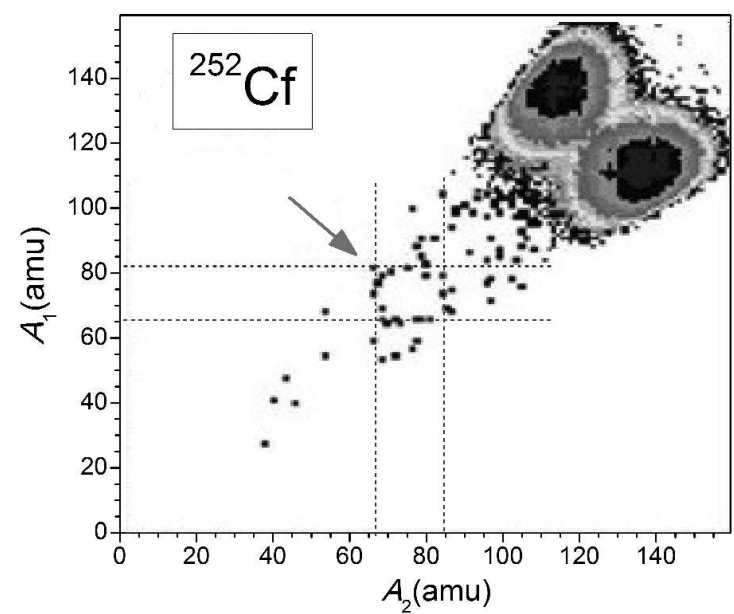

Fig. 36. Binary coincidences of fragments in the spontaneous ternary decay (FFF) of ${ }^{252} \mathrm{Cf}(\mathrm{sf})$ selected for the symmetric decay, from the inclusive data, measured in Ref. 32. The outer fragments $\left(A_{1}, A_{2}\right.$, with masses $\left.M_{1}, M_{2}\right)$ are selected with the condition for the velocities and the momenta $P_{i}:\left(V_{1} \approx V_{2}\right.$, and $\left.P_{1} \approx P_{2}\right)$. Remnants of the binary fragments in coincidences are seen. The region of the FFF-decays is marked. Missing fragments $\left(A_{3}\right)$ here are again isotopes of nuclei with $\left(Z_{3}=30\right.$ - 36). Scattered points above this region are true coincidences of other symmetric ternary decays, from Ref. 33].

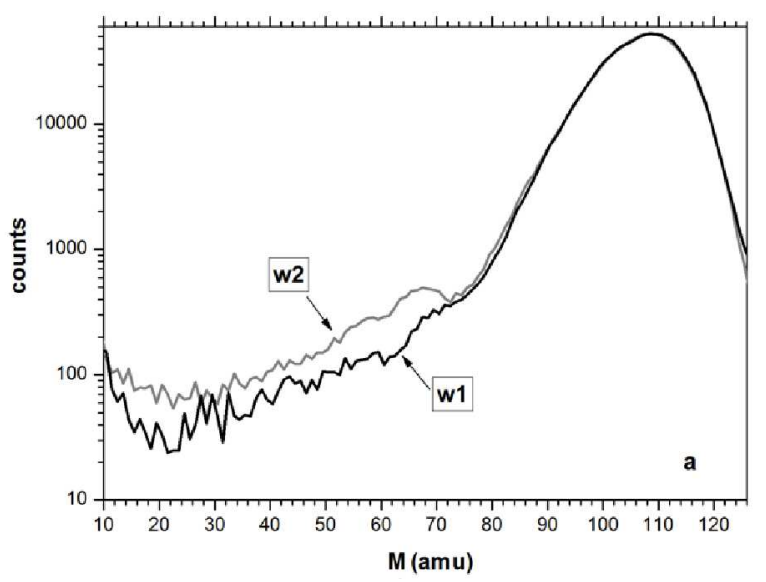

Fig. 37. Extraction of the yield from the data of Ref. 32,63]: the "Mo-Ar"-mode. The two different mass spectra, obtained from the two arms (arm1 and arm2). The data are projected to the $A_{1}$ axis for arm 1 and arm2 measured in Ref. 32 with binary coincidences.

events in the experiment. A quite equivalent decay pattern is observed in the ternary fission in the ${ }^{236} \mathrm{U}(\mathrm{n}, \mathrm{fff})$ reaction, where the dominant decays are connected to the proton-shells. We show in Fig 13 the PES for this case, the observed decays (Ref. [37]) are analogous to the spontaneous fission in ${ }^{252} \mathrm{Cf}(\mathrm{sf})$. Several favored fragment combinations are observed, including the symmetric decay discussed in Refs. 37, 38.

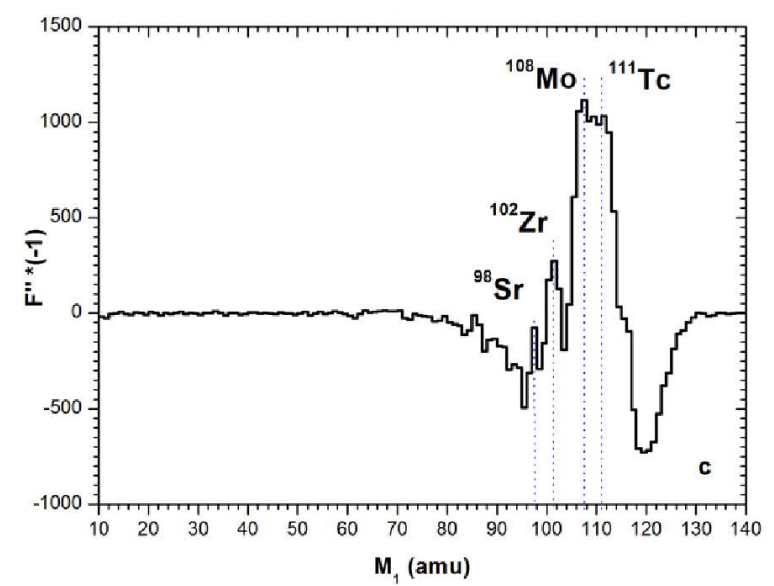

Fig. 38. The derivative of the difference of the two spectra shown in Fig 37 , events in the region of ${ }^{106} \mathrm{Mo}$ appear. The complementary missing fragments, $\left(A_{3}\right)$, here are isotopes of Ar with $\left(Z_{3}=18\right)$.

For ${ }^{252} \mathrm{Cf}(\mathrm{sf})$ we can select this symmetric decay mode from the overall data of Ref. [32,33] by choosing conditions on the momenta (velocities) of $Z_{2}$ and $Z_{1}$, with $\left(P_{1}=\right.$ $\left.P_{2}\right)$, see Fig. 36. This choice produces an rectangular twodimensional field of the experimental binary coincident events in the favored region (similar result will be obtained by the reflection of the labels), see also Fig. 7 .

An unexpected favored ternary decay mode is connected to the charge $Z=18$ seen in the PES of both cases ${ }^{252} \mathrm{Cf}(\mathrm{sf})$ and ${ }^{236} \mathrm{U}(\mathrm{n}$,fff) as a blue dip. A special procedure had to be chosen to obtain the yield of this "Mo-mode". The original spectra show only a small difference in the two detectors. Making a first derivative of this difference we observe a strong peak for the mass region of $A_{3}=102$ - 110 (shown in Fig 38).

\section{Summary}

In this survey we have discussed several theoretical approaches to the ternary fission decay of heavy nuclei. From principal considerations we conclude that the decay will be sequential. This conclusion comes from arguments with the phase space, this is generally much smaller (three decay vectors) for spontaneous three-body final channels than in a sequence of two two-body decays. In the latter two barriers appear in the respective two-body channels. For this case the kinetic energies have been calculated for the three fragments in a collinear geometry. In this case the central (smaller) fragment attains energies close to zero (0), giving a binary coincidence event with a missing mass. Thus in the experiments discussed, the central fragments are generally lost by absorption in the target and/or in the target backing. Some detailed predictions of the sequential ternary decay are consistently described in the theoretical analysis in two of the studied cases.

From the two experimental cases of the observed ternary fission in heavy nuclei with $\mathrm{U}$ and $\mathrm{Cf}$, which have large $Q$ - 
values for a split into three nuclear fragments with comparable masses studied in the present survey, we can conclude that the appearance of deformed shells in the total system and in the fragments (for certain proton and neutron numbers) turns out to be decisive for the occurrence of true ternary fission at low excitation energy. In all cases the fragments with $Z=50$ (Tin isotopes) dominate the outcome of the decay. The ternary decay in ${ }^{252} \mathrm{Cf}(\mathrm{sf})$ is a unique case, because of deformed shells and because in all three fragments closed shells for proton numbers $Z=$ 20,28 and 50 and for neutron numbers $(20,28,50,126)$ appear, these induce the decisive lowering of the second barrier. In the case of ${ }^{252} \mathrm{Cf}(\mathrm{sf})$ the shells in the lighter fragments appear with, $Z=8,20,28$. For the discussion of the decay modes the potential energy surfaces (PES's) are shown.

In the survey of the experimental data, we notice that for completely independent experimental setups with binary coincidences for two fragments (for ${ }^{252} \mathrm{Cf}(\mathrm{sf})$ ) and for the completely different physical case, ${ }^{235} \mathrm{U}$ (n,fff) with a smaller total mass and charge, we have the same physical phenomenon, the observation of the missing mass in the coincidence of two binary fragments, with a comparable mass for the third fragment. The latter $\left({ }^{236} \mathrm{U}(\mathrm{n}, \mathrm{fff})\right)$ case shows the same feature with different and smaller (as expected) ternary fragment masses. The decays are observed with dominant fragments with $Z=50$, which are consistent with the independent and different theoretical predictions, mainly based on the PES's. Based on the PES's different ternary decays, multi-modal decays, can be predicted. These have been extracted by using corresponding conditions (gates in the experimental data) in the analysis of the experimental data. In the analysis of the various decay properties we find that the models based on preformed clusters show deficiencies, which are only partially removed by introducing deformation in the fragments. The approaches with three preformed clusters allow the calculation of the PES's, which determine the phase space for the decay after scission, once the fragments have been formed. In an detailed analysis of ternary fragmentations, based on an approach with three clusters by Holmval et al. 23] it is actually shown that in the case of ${ }^{252} \mathrm{Cf}(\mathrm{sf})$ ternary fragmentation is not possible if nuclear interaction between fragment and deformed shell effects are not considered.

The approach of Karpov 28, based on the three-center shell model, with strongly deformed shapes contains the maximum of necessary macroscopic and microscopic features and gives the best description for the dynamics with the formation of two necks, and finally of the three fragments. This approach can be connected to the concept of hyper-deformation with strongly deformed (elongated) shapes, which show the path towards ternary decays. The present work shows the uniqueness of the case of ${ }^{252} \mathrm{Cf}(\mathrm{sf})$, namely that the ternary fragmentation occurs due the shell effects in the decaying nucleus and in all three fragments. Already in the dominant case of fragments with $Z=50$, the decay barriers are high in the liquid drop potential energies, fission can only be observed due the shell effects in the fragments with $Z=20,28$ and $Z=50$. For the case of ${ }^{235} \mathrm{U}(\mathrm{n}, \mathrm{fff})$ the excitation energy brought in by the neutron capture is decisive to induce ternary fragmentation.

From our analysis we conclude that the CCT-decay observed in Ref. [32, the collinear cluster tripartition can be considered as a manifestation of hyper-deformation in heavy deformed nuclei.

\section{Acknowledgments}

We thank A. Karpov, D. Kamanin and Y. Pyatkov for many useful discussions and preparation of figures.

\section{Dedication}

The authors dedicate this survey to the memory of two colleges, who passed away in recent years, Walther Greiner (passed away in 2016) of Frankfurt (Germany) and Valerij Zagrebaev (passed away in 2015) of FLNR in Dubna, (Russian Federation) (e.g. Refs. [41,57]). They had contributed many ideas and important steps for the development of various aspects in the physics of true ternary fission.

\section{References}

1. O. Hahn and F. Strassman Naturwissentschaften, 27 p11, (1939).

2. E. Fermi Nature Vol. 133898 (1934).

3. L. Meitner and O. Frisch Nature Vol. 143 239-240 (1939).

4. N. Bohr and J. A. Wheeler Phys. Rev. 56426 (1939).

5. Fifty Years research in Nuclear Fisson, Nucl. Phys. A 502 1c-637c (1989) Eds. D. Hilscher, H.J. Krappe and W. von Oertzen.

6. Proceedings, ed. by L.W. Drabchinski, Fission of Nuclei 50 Years, Chlopin Radium Institute, Leningrad, Vol. 1 and 2 (1992).

7. 50 years with nuclear fission, National Academy of Science, Washington D.C. USA, and National Institute of Standards and Technology, Gaithersburg, Maryland USA (1989).

8. V. M. Strutinsky Nucl. Phys. A 502 67c-84c (1989).

9. V. M. Strutinsky Nucl. Phys. 46639 (1963).

10. H.J. Krappe and k. Pomorski, Theory of Nuclear Fission: A Textbook, Springer, Berlin Vol.838 (2012) .

11. C. Wagemans $\mathrm{C}$ (ed) The nuclear fission process, (CRC Press Inc., Boca Raton, Florida USA) (1991).

12. F. Gönnenwein Nucl. Phys. A 734213 (2004).

13. W. J. Swiatecki, Second UN Int. Conf. on the Peaceful Uses of Atomic Energy, Geneva, Switzerland, 1958 (United Nations, 1958), p. 651.

14. D. Diehl and W. Greiner Nucl. Phys. A 22929 (1974).

15. M. A. Riley et al., Phys. Scr. 912015 (1992).

16. S.M. Polikanov, V.A. Druin, V.A. Karnaukhov, V.L. Mikheev, A.A. Pleve, N.K. Skobelev, V.G. Subbotin, G.M. Ter-Akopjan, V.A. Fomichev Zh. Eksperim. Theor. Fiz., 42 (1962), p. 1464 transl. Soviet Phys. JETP, 15 (1962) p. 1016 
17. S.M. Polikanov and G. Sletten Nucl. Phys. A 151656 (1970).

18. B. Singh, R. Zywina, and R. Firestone, Table of Superdeformed Nuclear Bands and Fission Isomers, 3rdEdition, Nuclear Data Sheets, 97 241, (2002).

19. V. Metag, D. Habs. H.J. Specht Phys. Rep. 65 1-41 (1980).

20. M. Brack,J. Damgard, A.S. Jensen, H. C. Pauli, V. M. Strutinsky,C.Y. Wong Rev. Mod. Phys. 44320 (1972)

21. M. Csatlos, A. Krasnahorkay, P.G. Thirolf et al., Phys. Letters B 615213 (2005).

22. K. R. Vijayaraghavan, M. Balasubramaniam and W. von Oertzen, Phys.Rev. C 900246001 (2014).

23. P. Holmvall, U. Köster, A. Heinz, and T. Nilsson Phys.Rev. C 95, 014602 (2017)

24. G. Royer et al., J. Phys. G: Nucl. Part. Phys. 182015 (1992) and Private communication.

25. E. Garrido et al. Nucl. Phys. A 74827 (2005) and Nucl. Phys. A 76639 (2006)

26. Yu. V. Pyatkov et al. Phys. Rev. C 96064606 (2017).

27. U. Brosa et al., Phys. Rep. 197123002 (2016).

28. A. V. Karpov Phys. Rev. C 94064615 (2016) and private communications.

29. J. Maruhn and W. Greiner Z. Phys. A 251431 (1972) and refs.

30. R. B. Tashkhodjaev, A. K. Nasirov and W. Scheid Eur. Phys. J. A 47136 (2011).

31. A. Wapstra, A. Audi and C. Thibault Nucl. Phys. A $\mathbf{7 2 9}$ 129 (2003).

32. Yu. V. Pyatkov et al. Eur. Phys. J. A 4529 (2010).

33. Yu. V. Pyatkov et al. Eur. Phys. J. A 4894 (2012).

34. C.Y. Wong Phys. Rev. Lett. 31766 (1973).

35. B. Fornal, R. Broda, et al. Eur. Phys. J. A 7147 (2000).

36. G. Sorlin Nucl. Phys. A 685 (2001).

37. W.von Oertzen and A. K. Nasirov Phys. Letters B 734, 234 (2014).

38. W.von Oertzen and A. K. Nasirov Phys. Letters B $\mathbf{7 4 6}$, 223(2015).

39. Yu.Z. Oganessian, et al. Phys. Rev. C 69, 054607 (2004).

40. M. Balasubramaniam K. R. Vijayaraghavan and K. Manimaran Phys.Rev. C 93014601 (2016).

41. V. I. Zagrebaev, A. V. Karpov and W. Greiner Phys. Rev. C 81, 044608 (2010).

42. J. Wilczynski et al. Phys. Rev. C 81067604 (2010).

43. P. Moeller, J. R. Nix, W. D. Meyers and W. J. Swiatecki, Atomic and Nuclear Data Tables Vol. 59 (1995) 185

44. K. Manimaran Phys. Rev. C 83064615 (2011).

45. R. B. Tashkhodjaev, A. I. Muminov, A. K. Nasirov, W. von Oertzen and Yonseok Oh Phys. Rev. C 91054612 (2015).

46. R. B. Tashkhodjaev, A. K. Nasirov and E. Kh. Alpomeshev Phys. Rev. C 94054614 (2016).

47. A. K. Nasirov, R. B. Tashkhodjaev, and W. von Oertzen Eur. Phys. J. A 52135 (2016).

48. K. R. Vijayaraghavan et al. J. Phys. G: Nucl. Part. Phys. G 46025103 (2019).

49. K. Manimaran et al. Phys. Rev. C 83034609 (2011).

50. K. R. Vijayaraghavan, W. von Oertzen and M. Balasubramaniam Eur. Phys. J. A 4827 (2012).

51. P. Schall et al. Phys. Lett. B 191339 (1987).

52. T. V. Chuvilskaya and Yu. M. Tchuvilsky Phys. Rev. C 99 024301 (2019)

53. B. D. Wilkins, E.P. Steinberg and R.R. Chasman Phys. Rev. C 61832 (1976)

54. J. Blons Nucl. Phys. A 502 121c-140c (1989)
55. D. Rochman et al. Nucl. Phys. A 7353 (2004).

56. D. N. Poenaru, R.A. Gherghescu and W. Greiner Nucl. Phys. A 747182 (2005).

57. V.I. Zagrebaev and W. Greiner 2010 in Lecture Notes in Physics 818 267-315 Clusters in Nuclei, Vol. 1 Beck C (ed.), Chapter 7, Springer-Verlag (Heidelberg, Berlin).

58. G. Adamian, N. Antonenko and W. Scheid in Lecture Notes in Physics 848 165-228 Clusters in Nuclei, Vol.2, Beck C (ed), Springer-Verlag Berlin Heidelberg (2012).

59. D. Poenaru and W. Greiner in Lecture Notes in Physics 875 1-56 Clusters in Nuclei, Vol.1, Beck C (ed), SpringerVerlag Berlin Heidelberg 2010

60. H.G. Ortlepp et al. Nucl. Inst. Meth. A 40365 (1998).

61. A.N. Tyukavkin et al. Instrum. Exp. Tech. 52508 (2009).

62. W. von Oertzen, Y. V. Pyatkov and D. Kamanin Acta Phys. Polonica B 44447 (2013). Zakopane Conference

63. D. Kamanin and Y.V. Pyatkov in Lecture Notes in Physics Vol. 875 183-246 Clusters in Nuclei, Vol.3 Beck C (ed), Chapter 6. Springer(Heidelberg, Berlin) (2014).

64. A. K. Nasirov A K et al. 2014 Phys.Scripta 89, 054022 (2014). 